\title{
ANISOTROPIC PARABOLIC EQUATIONS WITH VARIABLE NONLINEARITY
}

\author{
S. Antontsev and S. Shmarev
}

Abstract

We study the Dirichlet problem for a class of nonlinear parabolic equations with nonstandard anisotropic growth conditions. Equations of this class generalize the evolutional $p(x, t)$-Laplacian. We prove theorems of existence and uniqueness of weak solutions in suitable Orlicz-Sobolev spaces, derive global and local in time $L^{\infty}$ bounds for the weak solutions.

\section{Introduction}

1.1. Statement of the problem and assumptions. Let $\Omega \subset \mathbb{R}^{n}$ be a bounded simple-connected domain and $0<T<\infty$. We consider the Dirichlet problem for the parabolic equation

$$
\left\{\begin{array}{l}
u_{t}-\sum_{i} \frac{d}{d x_{i}}\left[a_{i}(z, u)\left|D_{i} u\right|^{p_{i}(z)-2} D_{i} u+b_{i}(z, u)\right]+d(z, u)=0 \quad \text { in } Q_{T} \\
u=0 \text { on } \Gamma_{T}, u(x, 0)=u_{0}(x) \text { in } \Omega
\end{array}\right.
$$

where $z=(x, t) \in Q_{T} \equiv \Omega \times(0, T], \Gamma_{T}$ is the lateral boundary of the cylinder $Q_{T}, D_{i}$ denotes the partial derivative with respect to $x_{i}$ and

$$
\frac{d f(z, v)}{d x_{i}}=D_{i} f(z, v)+\frac{\partial f(z, v)}{\partial v} D_{i} v
$$

2000 Mathematics Subject Classification. 35K55, 35K65.

Key words. Nonlinear parabolic equation, nonstandard growth conditions, anisotropic nonlinearity.

The first author was partially supported by the research project POCI/MAT/61576/2004, FCT/MCES (Portugal).

The second author acknowledges the support of the research grants MTM-2004-05417 and MTM-2007-65088 (Spain). 
The coefficients $a_{i}(z, u), b_{i}(z, u)$ and $d(z, u)$ may depend on $z=(x, t)$, $u(z)$ and obey the following conditions:

$$
a_{i}(z, r), b_{i}(z, r), d(z, r) \text { are Carathéodory functions }
$$

(defined for $(z, r) \in \bar{Q}_{T} \times \mathbb{R}$, measurable in $z$ for every $r \in \mathbb{R}$, continuous in $r$ for a.a. $z \in Q_{T}$ ),

(1.3) $\forall(z, r) \in \bar{Q}_{T} \times \mathbb{R} \quad 0<a_{0} \leq a_{i}(z, r) \leq a_{1}<\infty, \quad a_{0}, a_{1}=$ const,

$$
\forall(z, r) \in Q_{T} \times \mathbb{R}
$$

(1.4) $\sum_{i}\left|b_{i}(z, r)\right|^{p_{i}^{\prime}(z)} \leq b_{0}|r|^{\lambda}+h_{b}(z), \quad p_{i}^{\prime}=\frac{p_{i}(z)}{p_{i}(z)-1}$,

$|d(z, r)| \leq d_{0}|r|^{\lambda-1}+h_{d}(z)$,

with positive constants $b, d_{0}, d_{1}, d_{2}, \lambda>1$, and

$$
h_{b}(z) \in L^{1}\left(Q_{T}\right), \quad h_{d}(z) \in L^{\lambda^{\prime}}\left(Q_{T}\right), \quad \lambda^{\prime}=\frac{\lambda}{\lambda-1} .
$$

The exponents $p_{i}(z)$ are given continuous in $Q_{T}$ functions such that

$$
p_{i}(z) \subset\left(p_{i}^{-}, p_{i}^{+}\right) \subseteq\left(p^{-}, p^{+}\right) \subset(1, \infty),
$$

with finite constants $p^{ \pm}, p_{i}^{ \pm}>1$. Moreover, it will be assumed throughout the paper that the exponents $p_{i}(z)$ are continuous in $Q_{T}$ with logarithmic module of continuity:

$$
\forall z, \zeta \in Q_{T},|z-\zeta|<1, \quad \sum_{i}\left|p_{i}(z)-p_{i}(\zeta)\right| \leq \omega(|z-\zeta|),
$$

where

$$
\varlimsup_{\tau \rightarrow 0^{+}} \omega(\tau) \ln \frac{1}{\tau}=C<+\infty .
$$

1.2. Physical motivation and previous work. The paper addresses the questions of existence and uniqueness of weak solutions to problem (1.1). The main feature of equation (1.1) is the variable character of nonlinearity which causes a gap between the monotonicity and coercivity conditions. Because of this gap, equations of the type (1.1) are usually termed equations with nonstandard growth conditions. Equation (1.1) can be viewed as a generalization of the evolutional $p$-Laplacian equation

$$
u_{t}=\operatorname{div}\left(|\nabla u|^{p-2} \nabla u\right)
$$

with the constant exponent of nonlinearity $p \in(1, \infty)$. During the last decades equation (1.8) was intensively studied and was casted for the role of a touchstone in the theory of nonlinear PDEs. There is extensive literature devoted to equation (1.8). We limit ourselves by referring 
here to monographs $[\mathbf{2 4}],[\mathbf{3 6}]$, papers $[\mathbf{5}],[\mathbf{9}],[\mathbf{2 0}],[\mathbf{2 9}]$ and the review paper $[\mathbf{3 0}]$ which provide an excellent insight to the theory of evolutional $p$-Laplacian equations.

PDEs with variable nonlinearity are very interesting from the purely mathematical point of view. On the other hand, their study is motivated by various applications where such equations appear in the most natural way. Equations of the type (1.1) and their elliptic counterparts appear in the mathematical descriptions of motions of the non-newtonian fluids [11], in particular, electro-rheological fluids which are characterized by their ability to change the mechanical properties under the influence of the exterior electro-magnetic field $[\mathbf{2 7}],[\mathbf{3 9}],[\mathbf{4 0}]$. Most of the known results concern the stationary models, see, e.g., [1], [2], [3]. Some properties of solutions of the system of modified nonstationary Navier-Stokes equations describing electro-rheological fluids are studied in [4]. Another important application is the image processing where the anisotropy and nonlinearity of the diffusion operator and convection terms are used to underline the borders of the distorted image and to eliminate the noise $[\mathbf{6}],[\mathbf{8}],[\mathbf{2 1}]$. Many of the frequently discussed schemes of image restoration lead to nonlinear elliptic and parabolic equations with linear growth in the diffusion operator; this situation corresponds to the case $p^{-}=1$ and is not discussed in the present paper.

To the best of our knowledge, the reported results on the solvability of parabolic equations of the type (1.1) concern the equations with linear growth at infinity whose solutions are understood as elements of the space $L^{2}\left(0, T ; B V(\Omega) \cap L^{2}(\Omega)\right)$, see, e.g., [7], [8], [21]. In our assumptions on the structure of the equation, the weak solutions possess better regularity and belong to Orlicz-Sobolev spaces $W^{1, p(\cdot)}\left(Q_{T}\right)$ (the rigorous definition is given in Section 2 below). Moreover, it is proved in [18] that the gradient of the solution to the evolutional $p(x, t)$-Laplacian satisfy the Meyer-type estimate: the gradient is integrable with the exponent $p(z)(1+\delta), \delta>0$, instead of $p(z)$ as is prompted by the equation. It is known also that the solutions of equation (1.1) may extinct in a finite time $[\mathbf{1 5}],[\mathbf{1 7}]$, a property typical for the solutions of the fast diffusion equation. In contrast to the case of the fast diffusion equation with constant exponents of nonlinearity, the variable nonlinearity makes that this property may persist even if the equation eventually transforms into the linear one. It is worth mentioning here the papers $[\mathbf{3 1}],[\mathbf{3 2}]$, [33] devoted to the study of similar effects in solutions of equations with singularly perturbed coefficients and exponents of nonlinearity. 
Parabolic equations with variable nonlinearity of the type

$$
u_{t}=\operatorname{div}\left(|u|^{\gamma(x, t)} \nabla u\right)+F(x, t, u, \nabla u)
$$

are studied in papers $[\mathbf{1 2}],[\mathbf{1 6}]$. This class of equations generalizes the famous porous media equation (PME) to the case of variable exponents of nonlinearity. It is shown in [12] that the weak solutions of this equation display many of the properties intrinsic to the solutions of PME. However, the methods used in the study of solvability of such equations are specific for the generalized PME and can not be directly applied to equations of the type (1.1) which are nonlinear with respect to $D_{i} u$.

Stationary counterparts of equation (1.1) and the generalized PME were studied by many authors. We refer here to [13], [14], [37] for a review of the relevant results.

1.3. Organization of the paper and description of results. The paper is organized as follows. In Section 2 we introduce the function spaces of Orlicz-Sobolev type and present a brief description of their main properties. In our conditions on the regularity of the data, the smooth functions are dense in these spaces, which allows us to construct a solution using the sequence of Galerkin's approximations.

The main existence result for problem (1.1) is stated in Theorem 3.1. We prove that problem (1.1) has at least one global weak solution if the growth conditions (1.4) and (1.6) are fulfilled with $2 \leq \lambda=\max \left\{2, p^{-}-\delta\right\}$ for some $\delta>0$. The assertion remains true if $\lambda=\max \left\{2, p^{-}\right\}$, but under the additional condition of smallness of the data $u_{0}, h_{d}$ and $h_{b}$ in the corresponding norms. The case $\lambda>\max \left\{2, p^{-}\right\}$is studied in Theorem 3.2. We show that in this range of exponents, and with the functions $h_{b}, h_{d}$ satisfying (1.5), problem (1.1) has a local in time solution if the parameters $\lambda, p^{-}$and $n$ are subject to the conditions

$$
\begin{aligned}
& \max \left\{2, p^{-}\right\}<\lambda<p^{-}\left(1+\frac{2}{n}\right), \\
& \max \left\{1, \frac{2 n}{2+n}\right\}<p^{-}, \\
& p^{-}\left(1+\frac{2}{n}\right)<\frac{n p^{-}}{n-p^{-}} \text {if } n>p^{-} .
\end{aligned}
$$

The proofs of these assertions do not require monotonicity of the term $d(z, u)$. The monotonicity of the diffusion part of the equation is used to prove the convergence of Galerkin's approximations. In Section 7 
we briefly discuss the possibility of extension of the existence results to the case of homogeneous Neumann boundary condition.

Section 4 is devoted to derivation of $L^{\infty}$ bounds for the solutions of problem (1.1). We assume that the functions $h_{d}, h_{b}$ are subject to the stronger restrictions

$$
\begin{aligned}
& |d(z, r)| \leq d_{0}|r|+h_{d}, \\
& \left|b_{i}(z, r)\right| \leq b_{0}|r|+h_{b},
\end{aligned}
$$

Under these assumptions we prove in Theorem 4.1 that the weak solutions of problem (1.1) are globally bounded. The growth restriction can be relaxed for the terms $d(z, u)$ of special form. Namely, if we assume that in the foregoing assumptions

$$
d(z, u)=d_{1}(z, u)|u|^{\sigma(z)-2} u+d_{2}(z, u)|u|^{\lambda-2} u+h_{d}
$$

with

$1<\lambda \leq \inf _{Q_{T}} \sigma(z)<M, \quad d_{1} \geq d_{01}=$ const $>0, \quad\left|d_{2}\right| \leq d_{2}=$ const $<\infty$, and that the inequality

$$
d_{01} R^{\sigma(z)-1}-d_{02} R^{\lambda-1}-b_{0} R-\sup _{Q_{T}} h_{d}(z)-\sup _{Q_{T}}\left|h_{b}(z)\right| \geq 0
$$

holds in $Q_{T}$ for some $R>0$, then the solutions of (1.1) are globally bounded. Moreover, once such a bound is established, we use it to prove the existence of a global weak solution applying Theorem 3.1. We finally drop conditions (1.9) and show the under assumptions (1.10) problem (1.1) admit a local bounded solution for every $\lambda \geq 1$.

Uniqueness of weak solutions is studied in Section 5. It is shown that the weak solution of problem (1.1) is unique if the function $u \mapsto d(z, u)$ is monotone increasing and

$$
\left|a_{i}(z, u)-a_{i}(z, v)\right| \leq \omega(|u-v|)
$$

with the module of continuity $\omega$ satisfying the condition

$$
\int_{\epsilon} \frac{d s}{\omega^{\alpha}(s)} \rightarrow \infty \quad \text { as } \epsilon \rightarrow 0 \quad \text { for some } 1<\alpha<\left(p^{+}\right)^{\prime}=\frac{p^{+}}{p^{+}-1} .
$$

If the omit the condition of monotonicity of $d(z, u)$, the uniqueness of weak solutions still can be proved but under stronger continuity and growth assumptions: $d(z, u)$ is Lipschitz-continuous with respect to $u$ and $\omega^{\alpha}(s)=C s^{2}$. In the proof of uniqueness we follow ideas of [10], $[\mathbf{1 4}],[\mathbf{2 2}],[\mathbf{2 3}]$ were similar arguments were applied to the study of elliptic equations with nonstandard growth conditions. 
In Section 6 we study the dependence of the regularity of solutions to problem (1.1) on the regularity properties of the exponents $p_{i}, a_{i}$ and $\sigma$ in the partial case when

$$
{ }_{i} \equiv a_{i}(z), \quad d(z, u)=c(z)|u|^{\sigma(z)-2} u-f(z), \quad c(z) \leq 0 .
$$

We show that if $u_{0} \in L^{\sigma(\cdot, 0)}(\Omega), D_{i} u_{0} \in L^{p_{i}(\cdot, 0)}(\Omega)$, and if the exponents $p_{i}$ and $\sigma$ are nonincreasing functions of $t$, then the solutions of problem (1.1) possess better regularity properties:

$$
\begin{gathered}
u_{t} \in L^{2}\left(Q_{T}\right), \quad|u|^{\sigma(z)},\left|D_{i} u\right|^{p_{i}(z)} \in L^{\infty}\left(0, T ; L^{1}(\Omega)\right), \\
\left|D_{i} u\right|^{p_{i}}|\ln | D_{i} u\left\|\left|p_{i t}\right|,|u|^{\sigma}|\ln | u\right\|\left|\sigma_{t}\right| \in L^{1}\left(Q_{T}\right) .
\end{gathered}
$$

In the concluding Section 7 we give certain extensions of the results to other classes of equations close to (1.1).

\section{The function spaces}

2.1. Spaces $L^{p(\cdot)}(\Omega)$ and $W_{0}^{1, p(\cdot)}(\Omega)$. The definitions of the function spaces used throughout the paper and a brief description of their properties follow $[\mathbf{2 5}],[\mathbf{2 6}],[\mathbf{3 4}],[\mathbf{3 8}]$. The further references can be found in the review papers $[\mathbf{2 8}],[\mathbf{4 1}]$. Let

(2.1) $\left\{\begin{array}{l}\Omega \subset \mathbb{R}^{n} \text { be a bounded domain, } \partial \Omega \text { be Lipschitz-continuous, } \\ p(x) \text { satisfy condition (1.7) of log-continuity. }\end{array}\right.$

By $L^{p(\cdot)}(\Omega)$ we denote the space of measurable functions $f(x)$ on $\Omega$ such that

$$
A_{p(\cdot)}(f)=\int_{\Omega}|f(x)|^{p(x)} d x<\infty .
$$

The space $L^{p(\cdot)}(\Omega)$ equipped with the norm

$$
\|f\|_{p(\cdot), \Omega} \equiv\|f\|_{L^{p(\cdot)(\Omega)}}=\inf \left\{\lambda>0: A_{p(\cdot)}(f / \lambda) \leq 1\right\}
$$

becomes a Banach space. The Banach space $W_{0}^{1, p(\cdot)}(\Omega)$ with $p(x) \in$ $\left[p^{-}, p^{+}\right] \subset(1, \infty)$ is defined by

$$
\left\{\begin{array}{l}
W_{0}^{1, p(\cdot)}(\Omega)=\left\{f \in L^{p(\cdot)}(\Omega):|\nabla f| \in L^{p(\cdot)}(\Omega), u=0 \text { on } \partial \Omega\right\} \\
\|u\|_{W_{0}^{1, p(\cdot)}(\Omega)}=\sum_{i}\left\|D_{i} u\right\|_{p(\cdot), \Omega}+\|u\|_{p(\cdot), \Omega}
\end{array}\right.
$$

An equivalent norm of $W_{0}^{1, p(\cdot)}$ is given by

$$
\|u\|_{W_{0}^{1, p(\cdot)}(\Omega)}=\sum_{i}\left\|D_{i} u\right\|_{p(\cdot), \Omega} .
$$


- If condition (2.1) is fulfilled, then $C_{0}^{\infty}(\Omega)$ is dense in $W_{0}^{1, p(\cdot)}(\Omega)$. The space $W_{0}^{1, p(\cdot)}(\Omega)$ can be defined then as the closure of $C_{0}^{\infty}(\Omega)$ with respect to the norm $(2.2)$ - see $[\mathbf{4 2}],[\mathbf{4 5}]$.

- The space $W^{1, p(\cdot)}(\Omega)$ is separable and reflexive provided that $p(x) \in$ $C^{0}(\bar{\Omega})$.

- Let

$$
1<q(x) \leq \sup _{\Omega} q(x)<\inf _{\Omega} p_{*}(x)
$$

with

$$
p_{*}(x)= \begin{cases}\frac{p(x) n}{n-p(x)} & \text { if } p(x)<n, \\ \infty & \text { if } p(x)>n .\end{cases}
$$

Then the embedding $W_{0}^{1, p(\cdot)}(\Omega) \hookrightarrow L^{q(\cdot)}(\Omega)$ is continuous and compact.

- It follows directly from the definition that

$$
\min \left(\|f\|_{p(\cdot)}^{p^{-}},\|f\|_{p(\cdot)}^{p^{+}}\right) \leq A_{p(\cdot)}(f) \leq \max \left(\|f\|_{p(\cdot)}^{p^{-}},\|f\|_{p(\cdot)}^{p^{+}}\right) .
$$

- Hölder's inequality. For all $f \in L^{p(\cdot)}(\Omega), g \in L^{p^{\prime}(\cdot)}(\Omega)$ with

$$
p(x) \in(1, \infty), \quad p^{\prime}=\frac{p}{p-1},
$$

the following inequality holds:

(2.4) $\int_{\Omega}|f g| d x \leq\left(\frac{1}{p^{-}}+\frac{1}{\left(p^{\prime}\right)^{-}}\right)\|f\|_{p(\cdot)}\|g\|_{p^{\prime}(\cdot)} \leq 2\|f\|_{p(\cdot)}\|g\|_{p^{\prime}(\cdot)}$.

- If conditions (2.1) are fulfilled, then there exists a constant $C>0$ such that

(2.5) $\forall f \in W_{0}^{1, p(\cdot)}(\Omega)\|f\|_{p(\cdot), \Omega} \leq C\|\nabla f\|_{p(\cdot), \Omega} \quad$ (Poincaré inequality).

2.2. Spaces $L^{p(\cdot, \cdot)}\left(Q_{T}\right)$ and anisotropic spaces $\mathrm{W}\left(Q_{T}\right)$. Let $p_{i}(z)$ satisfy conditions (1.6) and (1.7). For every fixed $t \in[0, T]$ we introduce 
the Banach space

$$
\begin{gathered}
\mathbf{V}_{t}(\Omega)=\left\{u(x): u(x) \in L^{2}(\Omega) \cap W_{0}^{1,1}(\Omega),\left|D_{i} u(x)\right|^{p(x, t)} \in L^{1}(\Omega)\right\} \\
\|u\|_{\mathbf{V}_{t}(\Omega)}=\|u\|_{2, \Omega}+\sum_{i}\left\|D_{i} u\right\|_{p_{i}(\cdot, t), \Omega},
\end{gathered}
$$

and denote by $\mathbf{V}_{t}^{\prime}(\Omega)$ its dual. For every $t \in[0, T]$ the inclusion

$$
\mathbf{V}_{t}(\Omega) \subset \mathbf{X}=W_{0}^{1, p^{-}}(\Omega) \cap L^{2}(\Omega)
$$

holds, which is why $\mathbf{V}_{t}(\Omega)$ is reflexive and separable as a closed subspace of $\mathbf{X}$.

By $\mathbf{W}\left(Q_{T}\right)$ we denote the Banach space

$$
\begin{gathered}
\mathbf{W}\left(Q_{T}\right)=\left\{u:[0, T] \mapsto \mathbf{V}_{t}(\Omega) \mid u \in L^{2}\left(Q_{T}\right),\right. \\
\left.\left|D_{i} u\right|^{p_{i}(z)} \in L^{1}\left(Q_{T}\right), u=0 \text { on } \Gamma_{T}\right\}, \\
\|u\|_{\mathbf{W}\left(Q_{T}\right)}=\sum_{i}\left\|D_{i} u\right\|_{p_{i}(\cdot), Q_{T}}+\|u\|_{2, Q_{T}} .
\end{gathered}
$$

$\mathbf{W}^{\prime}\left(Q_{T}\right)$ is the dual of $\mathbf{W}\left(Q_{T}\right)$ (the space of linear functionals over $\left.\mathbf{W}\left(Q_{T}\right)\right)$ :

$$
w \in \mathbf{W}^{\prime}\left(Q_{T}\right) \Longleftrightarrow\left\{\begin{array}{l}
w=w_{0}+\sum_{i=1}^{n} D_{i} w_{i}, \quad w_{0} \in L^{2}\left(Q_{T}\right), \quad w_{i} \in L^{p_{i}^{\prime}(\cdot)}\left(Q_{T}\right), \\
\forall \phi \in \mathbf{W}\left(Q_{T}\right) \quad\langle\langle w, \phi\rangle\rangle=\int_{Q_{T}}\left(w_{0} \phi+\sum_{i} w_{i} D_{i} \phi\right) d z .
\end{array}\right.
$$

The norm in $\mathbf{W}^{\prime}\left(Q_{T}\right)$ is defined by

$$
\|v\|_{\mathbf{W}^{\prime}\left(Q_{T}\right)}=\sup \left\{\langle\langle v, \phi\rangle\rangle \mid \phi \in \mathbf{W}\left(Q_{T}\right),\|\phi\|_{\mathbf{W}\left(Q_{T}\right)} \leq 1\right\} .
$$

Let $\mathbf{v}=\left(v_{1}, \ldots, v_{n}\right), \mathbf{p}(z)=\left(p_{1}(z), \ldots, p_{n}(z)\right)$, and

$$
A_{\mathbf{p}(\cdot), Q_{T}}(\mathbf{v})=\sum_{i=1}^{n} \int_{Q_{T}}\left|v_{i}\right|^{p_{i}(z)} d z .
$$

The following counterpart of (2.3) holds:

$$
\begin{array}{r}
\min \left\{\sum_{i}\left\|D_{i} u\right\|_{p_{i}(\cdot), Q_{T}}^{p^{+}}, \sum_{i}\left\|D_{i} u\right\|_{p_{i}(\cdot), Q_{T}}^{p^{-}}\right\} \leq A_{\mathbf{p}(\cdot), Q_{T}}(\nabla u) \\
\leq \max \left\{\sum_{i}\left\|D_{i} u\right\|_{p_{i}(\cdot), Q_{T}}^{p^{-}}, \sum_{i}\left\|D_{i} u\right\|_{p_{i}(\cdot), Q_{T}}^{p^{+}}\right\}
\end{array}
$$


Set

$$
\mathbf{V}_{+}(\Omega)=\left\{u(x)\left|u \in L^{2}(\Omega) \cap W_{0}^{1,1}(\Omega),\right| \nabla u \mid \in L^{p^{+}}(\Omega)\right\} .
$$

Since $\mathbf{V}_{+}(\Omega)$ is separable, it is a span of a countable set of linearly independent functions $\left\{\psi_{k}(x)\right\} \subset \mathbf{V}_{+}(\Omega)$. Without loss of generality, we may assume that this system forms an orthonormal basis of $L^{2}(\Omega)$.

Proposition 2.1. Let conditions (2.1) hold. Then the set $\left\{\psi_{k}\right\}$ is dense in $\mathbf{V}_{t}(\Omega)$ for every $t \in[0, T]$.

Proof: In our conditions on $\partial \Omega$ and $p_{i}$, for every $u \in \mathbf{V}_{t}(\Omega)$ there is a sequence $u_{\delta}(\cdot, t) \in C^{\infty}(\Omega)$ such that $\operatorname{supp} u_{\epsilon}(\cdot, t) \Subset \Omega$ and

$$
\left\|u-u_{\delta}\right\|_{\mathbf{V}_{t}(\Omega)} \rightarrow 0 \quad \text { as } \delta \rightarrow 0 .
$$

Such a sequence is obtained via convolution of $u$ with the Friedrics's mollifiers [45, Theorem 2.1]. Since $u_{\delta} \in C_{0}^{\infty}(\Omega) \subset \mathbf{V}_{+}(\Omega)$ and $\left\{\psi_{m}\right\}$ is dense in $\mathbf{V}_{+}(\Omega)$, one may choose constants $c_{m}$ such that

$$
u_{\delta}^{(k)} \equiv \sum_{m=1}^{k} c_{m} \psi_{m}(x) \rightarrow u_{\delta} \quad \text { strongly in } \mathbf{V}_{+}(\Omega) \text { as } \delta \rightarrow 0 .
$$

Given an arbitrary $\epsilon>0,\left\|u_{\delta}-u_{\delta}^{(k)}\right\|_{\mathbf{V}_{+}(\Omega)}<\epsilon$ for all $k \in \mathbb{N}$ from some $k(\epsilon)$ on. By $(2.4)$

$$
\left\|u_{\delta}-u_{\delta}^{(k)}\right\|_{\mathbf{v}_{t}(\Omega)} \leq C\left\|u_{\delta}-u_{\delta}^{(k)}\right\|_{\mathbf{v}_{+}(\Omega)} \leq C \epsilon
$$

with a constant $C=C\left(n,|\Omega|, p^{ \pm}, \sigma^{ \pm}\right)$independent of $\epsilon$. It follows now that for all sufficiently large $k$ and small $\delta$

$$
\left\|u-u_{\delta}^{(k)}\right\|_{\mathbf{V}_{t}(\Omega)} \leq\left\|u-u_{\delta}\right\|_{\mathbf{V}_{t}(\Omega)}+\left\|u_{\delta}-u_{\delta}^{(k)}\right\|_{\mathbf{V}_{t}(\Omega)}<2 \epsilon \quad \forall t \in[0, T] .
$$

Proposition 2.2. For every $u \in \mathbf{W}\left(Q_{T}\right)$ there is a sequence $\left\{d_{k}(t)\right\}$, $d_{k}(t) \in C^{1}[0, T]$, such that

$$
\left\|u-\sum_{k=1}^{m} d_{k}(t) \psi_{k}(x)\right\|_{\mathbf{W}\left(Q_{T}\right)} \rightarrow 0 \quad \text { as } m \rightarrow \infty .
$$

Proof: In view of Proposition 2.1, the assertion immediately follows because the functions $\sum_{k=1}^{m} d_{k}(t) \psi_{k}(x)$ are dense in $L^{p^{+}}\left(0, T ; W^{1, p^{+}}(\Omega)\right) \cap$ $L^{2}\left(0, T ; L^{2}(\Omega)\right)$.

Let $\rho$ be the Friedrics mollifying kernel

$$
\rho(s)=\left\{\begin{array}{ll}
\kappa \exp \left(-\frac{1}{1-|s|^{2}}\right) & \text { if }|s|<1, \\
0 & \text { if }|s|>1,
\end{array} \quad \kappa=\text { const : } \int_{\mathbb{R}^{n+1}} \rho(z) d z=1 .\right.
$$


Given a function $v \in L^{1}\left(Q_{T}\right)$, we extend it to the whole $\mathbb{R}^{n+1}$ by a function with compact support (keeping the same notation for the continued function) and then define

$$
v_{h}(z)=\int_{\mathbb{R}^{n+1}} v(s) \rho_{h}(z-s) d s \quad \text { with } \rho_{h}(s)=\frac{1}{h^{n+1}} \rho\left(\frac{s}{h}\right), \quad h>0 .
$$

Proposition 2.3. If $u \in \mathbf{W}\left(Q_{T}\right)$ with the exponents $p_{i}(z)$ satisfying (1.7), then

$$
\left\|u_{h}\right\|_{\mathbf{W}\left(Q_{T}\right)} \leq C\left(1+\|u\|_{\mathbf{W}\left(Q_{T}\right)}\right) \text { and }\left\|u_{h}-u\right\|_{\mathbf{W}\left(Q_{T}\right)} \rightarrow 0 \text { as } h \rightarrow 0 .
$$

Proposition 2.3 is an immediate byproduct of [45, Theorem 2.1].

Proposition 2.4. Let in the conditions of Proposition $2.3 u_{t} \in \mathbf{W}^{\prime}\left(Q_{T}\right)$. Then $\left(u_{h}\right)_{t} \in \mathbf{W}^{\prime}\left(Q_{T}\right)$, and for every $\psi \in \mathbf{W}\left(Q_{T}\right)$

$$
\left\langle\left\langle\left(u_{h}\right)_{t}, \psi\right\rangle\right\rangle \rightarrow\left\langle\left\langle u_{t}, \psi\right\rangle\right\rangle \quad \text { as } h \rightarrow 0 .
$$

Proof: By the definition of $\mathbf{W}^{\prime}\left(Q_{T}\right)$ there exist $\phi_{0} \in L^{2}\left(Q_{T}\right), \phi_{i} \in$ $L^{p_{i}^{\prime}(\cdot)}\left(Q_{T}\right)$ such that $\left\langle\left\langle u_{t}, \psi\right\rangle\right\rangle=\left(\phi_{0}, \psi\right)_{2, Q_{T}}+\sum_{i}\left(\phi_{i}, D_{i} \psi\right)_{2, Q_{T}}, \forall \psi \in$ $\mathbf{W}\left(Q_{T}\right)$. It follows that

$$
\begin{aligned}
\left\langle\left\langle\left(u_{h}\right)_{t}, \psi\right\rangle\right\rangle & =\int_{Q_{T}}\left(u_{t}\right)_{h} \psi d z=\int_{Q_{T}} u_{t} \psi_{h} d z \\
& =\int_{Q_{T}}\left(\phi_{0} \psi_{h}+\sum_{i} \phi_{i} D_{i} \psi_{h}\right) d z \\
& =\int_{Q_{T}}\left(\left(\phi_{0}\right)_{h} \psi+\sum_{i}\left(\phi_{i}\right)_{h} D_{i} \psi\right) d z \rightarrow\left\langle\left\langle u_{t}, \psi\right\rangle\right\rangle \quad \text { as } h \rightarrow 0
\end{aligned}
$$

by virtue of Proposition 2.3.

Proposition 2.5 (Integration by parts). Let $v, w \in \mathbf{W}\left(Q_{T}\right)$ and $v_{t}, w_{t} \in$ $\mathbf{W}^{\prime}\left(Q_{T}\right)$ with the exponents $p_{i}(z)$ satisfying (1.7). Then

$\forall$ a.e. $t_{1}, t_{2} \in(0, T) \quad \int_{t_{1}}^{t_{2}} \int_{\Omega} v w_{t} d z+\int_{t_{1}}^{t_{2}} \int_{\Omega} v_{t} w d z=\left.\int_{\Omega} v w d x\right|_{t=t_{1}} ^{t=t_{2}}$. 
Proof: Let $t_{1}<t_{2}$. Take

$$
\chi_{k}(t)= \begin{cases}0 & \text { for } t \leq t_{1} \\ k\left(t-t_{1}\right) & \text { for } t_{1} \leq t \leq t_{1}+\frac{1}{k}, \\ 1 & \text { for } t_{1}+\frac{1}{k} \leq t \leq t_{2}-\frac{1}{k}, \\ k\left(t_{2}-t\right) & \text { for } t_{2}-\frac{1}{k} \leq t \leq t_{2}, \\ 0 & \text { for } t \geq t_{2} .\end{cases}
$$

For every $k \in \mathbb{N}$ and $h>0$

$$
0=\int_{Q_{T}}\left(v_{h} w_{h} \chi_{k}\right)_{t} d z \equiv \int_{Q_{T}}\left(v_{h} w_{h}\right)_{t} \chi_{k} d z-\left.k \int_{\theta-\frac{1}{k}}^{\theta} \int_{\Omega} v_{h} w_{h} d z\right|_{\theta=t_{1}} ^{\theta=t_{2}} .
$$

The last two integrals on the right-hand side exist because $v_{h}, w_{h} \in$ $L^{2}\left(Q_{T}\right)$. Letting $h \rightarrow 0$, we obtain the equality

$\lim _{h \rightarrow 0} \int_{Q_{T}}\left(v_{h}\left(w_{h}\right)_{t}+\left(v_{h}\right)_{t} w_{h}\right) \chi_{k}(t) d z=k \int_{t_{2}-\frac{1}{k}}^{t_{2}} \int_{\Omega} v w d z-k \int_{t_{1}}^{t_{1}+\frac{1}{k}} \int_{\Omega} v w d z$.

According to Propositions 2.3 and $2.4 v_{h} \rightarrow v$ in $\mathbf{W}\left(Q_{T}\right),\left(w_{h}\right)_{t}=$ $\left(w_{t}\right)_{h} \rightarrow w_{t}$ weakly in $\mathbf{W}^{\prime}\left(Q_{T}\right)$ as $h \rightarrow 0$, and $\|v\|_{\mathbf{W}},\left\|\left(w_{h}\right)_{t}\right\|_{\mathbf{W}^{\prime}}$ are uniformly bounded. It follows that

$$
\begin{aligned}
& \lim _{h \rightarrow 0} \int_{Q_{T}} v_{h}\left(w_{h}\right)_{t} \chi_{k}(t) d z=\lim _{h \rightarrow 0} \int_{Q_{T}}\left(v_{h}-v\right)\left(w_{h}\right)_{t} \chi_{k}(t) d z \\
+ & \lim _{h \rightarrow 0} \int_{Q_{T}} v\left(\left(w_{h}\right)_{t}-w_{t}\right) \chi_{k}(t) d z+\int_{Q_{T}} v w_{t} \chi_{k}(t) d z=\int_{Q_{T}} v w_{t} \chi_{k}(t) d z .
\end{aligned}
$$

In the same way we check that

$$
\lim _{h \rightarrow 0} \int_{Q_{T}}\left(v_{h}\right)_{t} w_{h} \chi_{k}(t) d z=\int_{Q_{T}} v w_{t} \chi_{k}(t) d z .
$$

By the Lebesgue differentiation theorem

$$
\forall \text { a.e. } \theta>0 \quad \lim _{k \rightarrow 0} k \int_{\theta-\frac{1}{k}}^{\theta}\left(\int_{\Omega} v w d x\right) d t=\int_{\Omega} v w d x,
$$

whence for almost every $t_{1}, t_{2} \in[0, T]$

$$
\begin{aligned}
\int_{t_{1}}^{t_{2}} \int_{\Omega}\left(v w_{t}+v_{t} w\right) d z & =\lim _{k \rightarrow \infty} \int_{Q_{T}}\left(v w_{t}+v_{t} w\right) \chi_{k}(t) d z \\
& =\left.\lim _{k \rightarrow \infty} k \int_{\theta-\frac{1}{k}}^{\theta} \int_{\Omega} v w d x\right|_{\theta=t_{1}} ^{t=t_{2}}=\left.\int_{\Omega} v w d x\right|_{\theta=t_{1}} ^{t=t_{2}}
\end{aligned}
$$


Corollary 2.1. Let $u \in \mathbf{W}\left(Q_{T}\right)$ and $u_{t} \in \mathbf{W}^{\prime}\left(Q_{T}\right)$ with the exponents $p_{i}(z)$ satisfying (1.7). Then

$$
\forall \text { a.e. } t_{1}, t_{2} \in(0, T] \quad \int_{t_{1}}^{t_{2}} \int_{\Omega} u u_{t} d z=\left.\frac{1}{2}\|u\|_{2, \Omega}^{2}\right|_{t=t_{1}} ^{t=t_{2}} .
$$

\section{Existence theorems}

In this section we prove the existence of weak solutions to problem (1.1) under the general growth conditions (1.4). The solution of problem (1.1) is understood in the following sense.

Definition 3.1. A function $u(x, t) \in \mathbf{W}\left(Q_{T}\right) \cap L^{\infty}\left(0, T ; L^{2}(\Omega)\right)$ is called weak solution of problem (1.1) if for every test-function

$$
\zeta \in \mathbf{Z} \equiv\left\{\eta(z): \eta \in \mathbf{W}\left(Q_{T}\right) \cap L^{\infty}\left(0, T ; L^{2}(\Omega)\right), \eta_{t} \in \mathbf{W}^{\prime}\left(Q_{T}\right)\right\},
$$

and every $t_{1}, t_{2} \in[0, T]$ the following identity holds:

$$
\begin{array}{r}
\int_{t_{1}}^{t_{2}} \int_{\Omega}\left(u \zeta_{t}-\sum_{i}\left[a_{i}\left|D_{i} u\right|^{p_{i}-2} D_{i} u+b_{i}(z, u)\right] D_{i} \zeta-d(z, u) \zeta\right) d z \\
=\left.\int_{\Omega} u \zeta d x\right|_{t_{1}} ^{t_{2}}
\end{array}
$$

The following are the main results of this section.

Theorem 3.1. a) Let us assume that

1) the coefficients $a_{i}(z, r), b_{i}(z, r), d(z, r)$ satisfy conditions (1.2), (1.3), (1.4),

2) the exponents $p_{i}(z)$ satisfy (1.6) and (1.7),

3) the constant $\lambda$ satisfies the condition

$$
\lambda=\max \left\{2, p^{-}-\delta\right\} \quad \text { with some } \delta>0 .
$$

Then for every $u_{0} \in L^{2}(\Omega)$ problem (1.1) has at least one weak solution $u \in \mathbf{W}\left(Q_{T}\right)$ satisfying the estimate

(3.3) $\|u\|_{L^{\infty}\left(0, T ; L^{2}(\Omega)\right)}^{2}+\int_{Q_{T}} a_{0} \sum_{i}\left|D_{i} u\right|^{p_{i}} d z \leq M\left[\left\|u_{0}\right\|_{L^{2}(\Omega)}^{2}+K+1\right]$ with a constant $M$ independent of $u$ and $K=\left\|h_{b}\right\|_{1, Q_{T}}+\left\|h_{d}\right\|_{\lambda^{\prime}, Q_{T}}$. Moreover, $u_{t} \in \mathbf{W}^{\prime}\left(Q_{T}\right)$. 
b) The assertion remains true if (3.2) is substituted by the condition $\lambda=\max \left\{2, p^{-}\right\}$and the constant $b_{0}+d_{0}$ in (1.4) is appropriately small in comparison with $a_{0}$.

Theorem 3.2. Let us assume that in the conditions of Theorem 3.1 condition (3.2) is substituted by the following one:

(3.4) $\max \left\{2, p^{-}\right\}<\lambda<p^{-}\left(1+\frac{2}{n}\right)<\frac{n p^{-}}{n-p^{-}}, \quad \max \left\{1, \frac{2 n}{2+n}\right\}<p^{-}$.

Then there exists $T_{0}>0$, defined through $\left\|u_{0}\right\|_{L^{2} \Omega}^{2}+K$, such that problem (1.1) has at least one weak solution $u \in \mathbf{W}\left(Q_{T_{0}}\right)$ satisfying estimate (3.3) in $Q_{T_{0}}$. The weak solution exists globally in time if $\left\|u_{0}\right\|_{L^{2}(\Omega)}^{2}+K$ is sufficiently small.

\subsection{Proof of Theorems 3.1 and 3.2.}

3.1.1. Galerkin's approximations. A solution of problem (1.1) is constructed as the limit of the sequence of Galerkin's approximations. Let us define the operator

$$
\begin{array}{r}
\langle L v, \phi\rangle_{\Omega}=\int_{\Omega}\left(v_{t} \phi+\sum_{i=1}^{n}\left[a_{i}(z, v)\left|D_{i} v\right|^{p_{i}-2} D_{i} v+b_{i}(z, v)\right] D_{i} \phi+d(z, v) \phi\right) d x, \\
\phi \in \mathbf{V}_{t}(\Omega) .
\end{array}
$$

The approximate solutions to problem (1.1) are sought in the form

$$
u^{(m)}(z)=\sum_{k=1}^{m} c_{k}^{(m)}(t) \psi_{k}(x), \quad \psi_{k} \in \mathbf{V}_{+}(\Omega),
$$

where the coefficients $c_{k}^{(m)}(t)$ are defined from the relations

$$
\left\langle L u^{(m)}, \psi_{k}\right\rangle_{\Omega}=0, \quad k=1, \ldots, m .
$$

Equalities (3.5) generate the system of $m$ ordinary differential equations for the coefficients $c_{k}^{(m)}(t)$ :

$$
\left\{\begin{array}{l}
\left(c_{k}^{(m)}\right)^{\prime}=F_{k}\left(t, c_{1}^{(m)}(t), \ldots, c_{m}^{(m)}(t)\right), \\
c_{k}^{(m)}(0)=\int_{\Omega} u_{0}(x) \psi_{k} d x \quad k=1, \ldots, m .
\end{array}\right.
$$

If the coefficients $a_{i}, b_{i}, d$ and the exponents $p_{i}, \sigma$ satisfy the conditions of Theorem 3.1 a), the functions $F_{k}$ are continuous in all their arguments. 


\subsubsection{A priory estimates.}

Lemma 3.1. Let the conditions of Theorem 3.1 a) be fulfilled. Then for every $T<\infty$ and $m \in \mathbb{N}$ system (3.6) has a solution $\left\{c_{k}^{(m)}(t)\right\}_{k=1}^{m}$ on the interval $(0, T)$ and the corresponding function $u^{(m)}$ satisfies the estimate

$$
\begin{aligned}
&\left\|u^{(m)}(\cdot, t)\right\|_{L^{\infty}\left(0, T ; L^{2}(\Omega)\right)}^{2}+\int_{Q_{T}} a_{0} \sum_{i}\left|D_{i} u^{(m)}\right|^{p_{i}} d z \\
& \leq M\left[\left\|u_{0}\right\|_{L^{2}(\Omega)}^{2}+K+1\right]
\end{aligned}
$$

with the constants $M, K$ defined in the conditions of Theorem 3.1.

Proof: By Peano's Theorem, for every finite $m$ system (3.6) has a solution $c_{i}^{(m)}(t), i=1, \ldots, m$, on an interval $\left(0, T_{m}\right)$. Multiplying each of equalities (3.5) by $c_{k}^{(m)}(t)$ and summing over $k=1, \ldots, m$, we arrive at the relation

$$
\begin{aligned}
&\left.\frac{1}{2}\left\|u^{(m)}\right\|_{2, \Omega}^{2}\right|_{t=0} ^{t}+\int_{Q_{\tau}}\left(\sum_{i}\right. {\left[a_{i}\left(z, u^{(m)}\right)\left|D_{i} u^{(m)}\right|^{p_{i}}+b_{i}\left(z, u^{(m)}\right) D_{i} u^{(m)}\right] } \\
&\left.+d\left(z, u^{(m)}\right) u^{(m)}\right) d z=0, \quad \tau \in\left[0, T_{m}\right] .
\end{aligned}
$$

Using (1.3), (1.4) and applying Young's inequality, we estimate: $\forall \epsilon>0$

$$
\begin{aligned}
\left|b_{i}\left(z, u^{(m)}\right) D_{i} u^{(m)}\right| \leq & \in a_{0}\left|D_{i} u^{(m)}\right|^{p_{i}}+C\left|b_{i}\left(z, u^{(m)}\right)\right|^{p_{i}^{\prime}} \\
& \leq \epsilon a_{0}\left|D_{i} u^{(m)}\right|^{p_{i}}+C\left(b_{0}\left|u^{(m)}\right|^{\lambda}+\left|h_{b}\right|\right), \\
\left|d\left(z, u^{(m)}\right) u^{(m)}\right| \leq & \left(d_{0}+\epsilon_{d}\right)\left|u^{(m)}\right|^{\lambda}+C\left|h_{d}\right|^{\lambda^{\prime}}, \quad \epsilon_{d} \in(0,1),
\end{aligned}
$$

with a constant $C$ depending on $\epsilon, \epsilon_{d}, a_{0}, p^{-}, p^{+}$. Plugging (3.9)-(3.10) into (3.8), choosing $\epsilon$ sufficiently small and simplifying, we get the estimate

$$
\begin{aligned}
\left.\frac{1}{2}\left\|u^{(m)}\right\|_{2, \Omega}^{2}\right|_{t=0} ^{t=\tau} & +\int_{Q_{\tau}} a_{0} \sum_{i}\left|D_{i} u^{(m)}\right|^{p_{i}} d z \\
& \leq C \int_{Q_{\tau}}\left(\left(d_{0}+b_{0}+\epsilon_{d}\right)\left|u^{(m)}\right|^{\lambda}+\left|h_{b}\right|+\left|h_{d}\right|^{\lambda^{\prime}}\right) d z \\
& \leq C\left(d_{0}+b_{0}+\epsilon_{d}\right) \int_{Q_{\tau}}\left\|u^{(m)}\right\|^{\lambda} d z+C K .
\end{aligned}
$$


Let $\lambda=2$. Using Gronwall's inequality to estimate the function $\left\|u^{(m)}(\cdot, t)\right\|_{2, \Omega}^{2}$ and then reverting to (3.8), we obtain the required estimate $(3.7)$.

Let $2<\lambda=p^{-}-\delta$. This assumption yields the inequality $\lambda<\frac{n\left(p^{-}-\delta\right)}{n-p^{-}+\delta}$, which allows one to make use of the embedding theorem in Sobolev spaces:

$$
\left\|u^{(m)}(\cdot, t)\right\|_{\lambda, \Omega}^{\lambda} \leq C\left(\lambda, p^{-}, n\right)\left\|\nabla u^{(m)}\right\|_{p^{-}, \Omega}^{\lambda} .
$$

Applying now (2.3) and Young's inequality, we arrive at the inequality

$$
\begin{aligned}
\int_{\Omega}\left|u^{(m)}\right|^{\lambda} d x & \leq C\left(\int_{\Omega}\left|\nabla u^{(m)}\right|^{p^{-}} d x\right)^{\frac{\lambda}{p-}} \\
& \leq C\left[\left(\sum_{i} \int_{\Omega}\left|D_{i} u^{(m)}\right|^{p_{i}} d x\right)^{\frac{\lambda}{p^{-}}}+1\right] \\
& \leq \epsilon a_{0} \sum_{i} \int_{\Omega}\left|D_{i} u^{(m)}\right|^{p_{i}} d x+C\left(\epsilon, \delta, \Omega, a_{0}, p^{ \pm}\right) .
\end{aligned}
$$

Gathering these estimates with (3.8) and choosing $\epsilon$ appropriately small, we obtain the inequality

$$
\left.\frac{1}{2}\left\|u^{(m)}\right\|_{2, \Omega}^{2}\right|_{t=0} ^{t=\tau}+a_{0} \sum_{i} \int_{Q_{\tau}}\left|D_{i} u^{(m)}\right|^{p_{i}} d z \leq C(K+1) .
$$

The right-hand side of the obtained estimate does not depend on $m$, which is why the solution of system (3.6) can be continued to the maximal interval $[0, T]$.

Lemma 3.2. The assertion of Lemma 3.1 remains true for $\lambda=$ $\max \left\{2, p^{-}\right\}$, provided that the constant $b_{0}+d_{0}$ is sufficiently small in comparison with $a_{0}$.

Proof: We only have to study the case $\lambda=p^{-}$. Then the Poincaré inequality yields

$$
\int_{\Omega}\left|u^{(m)}\right|^{\lambda} d x \leq C \int_{\Omega}\left|\nabla u^{(m)}\right|^{p^{-}} d x, \quad C=C(n, \lambda) .
$$


Combining (3.11) with this inequality, we have that

$$
\text { (3.14) } \begin{aligned}
& \left.\frac{1}{2}\left\|u^{(m)}\right\|_{2, \Omega}^{2}\right|_{t=0} ^{t=\tau}+a_{0} \sum_{i} \int_{Q_{\tau}}\left|D_{i} u^{(m)}\right|^{p_{i}} d z \\
\leq & C\left(d_{0}+b_{0}+\epsilon_{d}\right) \sum_{i} \int_{Q_{\tau}}\left|D_{i} u^{(m)}\right|^{p_{i}} d z+C K \quad \text { with } \epsilon_{d} \in(0,1) .
\end{aligned}
$$

The conclusion follows if we claim that $C\left(b_{0}+d_{0}\right)<a_{0}$ and choose $\epsilon_{d}$ sufficiently small.

Lemma 3.3. Let condition (3.4) be fulfilled. Then there exists $T_{0}$, depending on $\left\|u_{0}\right\|_{2, \Omega}^{2}+K$, such that the assertion of Lemma 3.1 is true on every interval $[0, T]$ with $T<T_{0}$.

Proof: Instead of (3.12), we will make use of the interpolation inequality

$$
\left\|u^{(m)}\right\|_{\lambda, \Omega}^{\lambda} \leq C\left(\lambda, p^{-}, n\right)\left\|\nabla u^{(m)}\right\|_{p^{-}, \Omega}^{\theta \lambda}\left\|u^{(m)}\right\|_{2, \Omega}^{(1-\theta) \lambda}
$$

with the exponent

$$
\theta=\frac{\lambda-2}{\lambda} \frac{n p^{-}}{n p^{-}-2\left(n-p^{-}\right)} \in(0,1) .
$$

The inclusion $\theta \in(0,1)$ follows from condition (3.4):

$$
\frac{\theta \lambda}{p^{-}}=\frac{n(\lambda-2)}{n p^{-}-2\left(n-p^{-}\right)}<1 \Longleftrightarrow \lambda<p^{-}\left(1+\frac{2}{n}\right) .
$$

Applying (2.3) and Young's inequality we transform (3.15) to the form

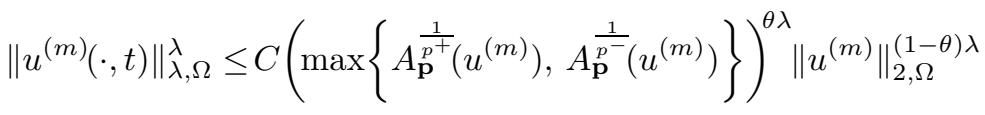

$$
\begin{aligned}
& \leq \epsilon A_{\mathbf{p}}\left(u^{(m)}\right)+C \max \left\{\left\|u^{(m)}\right\|_{2, \Omega}^{2 \gamma^{+}},\left\|u^{(m)}\right\|_{2, \Omega}^{2 \gamma^{-}}\right\}
\end{aligned}
$$

with the exponents

$$
\gamma^{ \pm}=\frac{(1-\theta) \lambda}{2} \frac{p^{ \pm}}{p^{ \pm}-\lambda \theta}>1 .
$$

Gathering (3.18) with (3.8)-(3.10) and choosing $\epsilon$ appropriately small, we have

$$
\begin{aligned}
\left.\frac{1}{2}\left\|u^{(m)}\right\|_{2, \Omega}^{2}\right|_{t=0} ^{t=\tau} & +\int_{Q_{\tau}} a_{0} \sum_{i}\left|D_{i} u^{(m)}\right|^{p_{i}} d z \\
& \leq C\left(\int_{0}^{\tau} \max \left\{\left\|u^{(m)}\right\|_{2, \Omega}^{\gamma^{+}},\left\|u^{(m)}\right\|_{2, \Omega}^{\gamma^{-}}\right\} d t+K\right) .
\end{aligned}
$$


Introducing the function

$$
Y(t)=\left\|u^{(m)}(\cdot, t)\right\|_{2, \Omega}^{2},
$$

we write the last inequality in the form

$$
\begin{gathered}
Y(t) \leq A+B \int_{0}^{t} \max \left\{Y^{\gamma^{+}}(\tau), Y^{\gamma^{-}}(\tau)\right\} d \tau \\
A=Y(0)+2 C K, \quad B=2 C
\end{gathered}
$$

The functions satisfying this inequality are bounded on the intervals $\left[0, t_{0}\right]$ with

$$
t_{0} \mathrm{e}^{(\gamma-1) B t_{0}}<\min \left\{\frac{1}{\left(\gamma^{+}-1\right) B A^{\frac{1}{\gamma^{+}-1}}}, \frac{1}{\left(\gamma^{-}-1\right) B A^{\frac{1}{\gamma^{-}-1}}}\right\}
$$

Since $t_{0} \rightarrow \infty$ as $A \rightarrow 0$, estimate (3.7) takes the form

$$
\left\|u^{(m)}(\cdot, t)\right\|_{2, \Omega}^{2}+\int_{Q t} a_{0} \sum_{i}\left|D_{i} u^{(m)}\right|^{p_{i}} d z \leq C\left(t_{0}\right)(1+K), \quad t \in\left[0, t_{0}\right] .
$$

3.1.3. Compactness and passage to the limit. Throughout this subsection we assume that $T$ satisfies the conditions of Lemmas 3.1, 3.2, and 3.3. Let us show that the constructed sequence $\left\{u^{(m)}\right\}$ is convergent.

Lemma 3.4. Under the conditions of Lemma 3.1, for every $m \in \mathbb{N}$ we have $u_{t}^{(m)} \in \mathbf{W}^{\prime}\left(Q_{T}\right)$ and

$$
\left\|u_{t}^{(m)}\right\|_{\mathbf{W}^{\prime}\left(Q_{T}\right)} \leq C^{\prime}\left[1+K+\left\|u_{0}\right\|_{2, \Omega}^{2}\right]
$$

Proof: Let

$$
\mathbf{Z}_{m}=\left\{\eta(x, t) \mid \eta=\sum_{k=1}^{m} d_{k}(t) \psi_{k}(x), d_{k}(t) \in C^{1}(0, T)\right\} \subset \mathbf{Z}
$$

be a subspace of the set of admissible test-functions. Take a function

$$
\phi=\sum_{i=1}^{m} \phi_{k}(t) \psi_{k}(x) \in \mathbf{Z}_{m} \quad \text { with } \phi_{k}(0)=\phi_{k}(T)=0 .
$$


By the definition of $u^{(m)}$ (see (3.6))

$$
\begin{aligned}
\int_{Q_{T}} u_{t}^{(m)} \phi d z= & -\int_{Q_{T}} u^{(m)} \phi_{t} d z \\
= & \sum_{i=1}^{n} \int_{Q_{T}}\left[a_{i}\left(z, u^{(m)}\right)\left|D_{i} u^{(m)}\right|^{p_{i}-2} D_{i} u^{(m)}+b_{i}\left(z, u^{(m)}\right)\right] D_{i} \phi d z \\
& +\int_{Q_{T}} d\left(z, u^{(m)}\right) \phi d z
\end{aligned}
$$

Using (1.4) and (3.7), we may estimate the right-side of this equality as follows:

$$
\begin{aligned}
& \left.\left|\int_{Q_{T}} \sum_{i=1}^{n} a_{i}\left(z, u^{(m)}\right)\right| D_{i} u^{(m)}\right|^{p_{i}-2} D_{i} u^{(m)} D_{i} \phi d z \mid \\
& \quad \leq C \sum_{i=1}^{n}\left\|\left|D_{i} u^{(m)}\right|^{p_{i}-1}\right\|_{p_{i}^{\prime}, Q_{T}}\left\|D_{i} \phi\right\|_{p_{i}, Q_{T}} \leq \widetilde{C} \sum_{i=1}^{n}\left\|D_{i} \phi\right\|_{p_{i}, Q_{T}}, \\
& \left|\int_{Q_{T}} \sum_{i=1}^{n} b_{i}\left(z, u^{(m)}\right) D_{i} \phi d z\right| \\
& \quad \leq C \sum_{i=1}^{n}\left\|b_{i}\right\|_{p_{i}^{\prime}, Q_{T}}\left\|D_{i} \phi\right\|_{p_{i}, Q_{T}} \leq \widetilde{C} \sum_{i=1}^{n}\left\|D_{i} \phi\right\|_{p_{i}, Q_{T}}, \\
& \quad \leq\left[\int_{Q_{T}} d\left(z, u^{(m)}\right) \phi d z \mid \leq\|d\|_{\lambda^{\prime}, Q_{T}}\|\phi\|_{\lambda, Q_{T}}\right. \\
& \quad \leq\left[\left(d_{0}, \lambda\right)\left\|\left|u^{(m)}\right|^{\lambda-1}\right\|_{\lambda^{\prime}, Q_{T}}+\left\|h_{d}\right\|_{\lambda^{\prime}, Q_{T}}\right]\|\phi\|_{\lambda, Q_{T}} \\
& \quad \leq C^{\prime}\left[1+\left\|u^{(m)}\right\| \mathbf{w}\left(Q_{T}\right)\right]\|\phi\|_{\lambda, Q_{T}}
\end{aligned}
$$

with the constants $C^{\prime}$ and $\widetilde{C}$ independent of $m$. It follows that for every $\phi \in \mathbf{Z}_{m}$

$$
\left|\int_{Q_{T}} u_{t}^{(m)} \phi d z\right| \leq C\left[1+\left\|u^{(m)}\right\|_{\mathbf{W}\left(Q_{T}\right)}\right]\|\phi\|_{\mathbf{W}\left(Q_{T}\right)} .
$$


The following inclusions hold:

$$
\left\{\begin{array}{l}
u^{(m)} \in \mathbf{W}\left(Q_{T}\right) \subseteq L^{p^{-}}\left(0, T ; W_{0}^{1, p^{-}}(\Omega)\right), \\
u_{t}^{(m)} \in \mathbf{W}^{\prime}\left(Q_{T}\right) \subseteq L^{\frac{p^{+}}{p^{+}-1}}\left(0, T ; \mathbf{V}_{+}^{\prime}(\Omega)\right) \\
W_{0}^{1, p^{-}}(\Omega) \subset L^{2}(\Omega) \subset \mathbf{V}_{+}^{\prime}(\Omega)
\end{array}\right.
$$

It follows that the sequence $\left\{u^{(m)}\right\}$ contains a subsequence strongly convergent in $L^{q}\left(Q_{T}\right)$ with some $q>1$ [43]. This subsequence contains a subsequence which converges to $u$ a.e. in $Q_{T}$ (see, e.g., [35, Theorem 2.8.1]). These conclusions together with the uniform in $m$ estimates (3.7) allow one to extract from the sequence $\left\{u^{(m)}\right\}$ a subsequence (for the sake of simplicity we assume that it merely coincides with the whole of the sequence) such that

$(3.21)$

$$
\begin{cases}u^{(m)} \rightarrow u & \text { weakly in } \mathbf{W}\left(Q_{T}\right) \\ u_{t}^{(m)} \rightarrow u_{t} & \text { and strongly in } L^{q}\left(Q_{T}\right), \\ u^{(m)} \rightarrow u & \text { weakly in } \mathbf{W}^{\prime}\left(Q_{T}\right), \\ d\left(z, u^{(m)}\right) \rightarrow d(z, u) & \text { a.e. in } Q_{T}, \\ b_{i}\left(z, u^{(m)}\right) \rightarrow b_{i}(z, u) & \text { strongly in } L^{\lambda^{\prime}}\left(Q_{T}\right), \\ a_{i}\left(z, u^{(m)}\right)\left|D_{i} u^{(m)}\right|^{p_{i}(z)-2} D_{i} u^{(m)} \rightarrow A_{i}(z) & \text { strongly in } L^{p_{i}^{\prime}(\cdot)}\left(Q_{T}\right),\end{cases}
$$

for some functions

$$
u \in \mathbf{W}\left(Q_{T}\right), \quad A_{i}(z) \in L^{p_{i}^{\prime}(\cdot)}\left(Q_{T}\right) .
$$

By the method of construction, each of the functions $u^{(m)}$ satisfies identity (3.1) with the test-function $\eta \in \mathbf{Z}_{m}$. Let us fix an arbitrary $m \in \mathbb{N}$. Then for every $s \leq m$ and $\eta \in \mathbf{Z}_{s}$

$$
\begin{gathered}
\left.\int_{\Omega} u^{(m)} \eta d x\right|_{\tau=0} ^{\tau=T} \\
-\int_{Q_{T}}\left[u^{(m)} \eta_{t}-\sum_{i}\left(a_{i}\left|D_{i} u^{(m)}\right|^{p_{i}-2} D_{i} u^{(m)}+b_{i}\right) D_{i} \eta+d \eta\right] d x d t=0 .
\end{gathered}
$$


Letting $m \rightarrow \infty$ and using (3.21) we conclude that $\forall \eta \in \mathbf{Z}_{s}$

$$
\begin{aligned}
(3.22) & -\left.\int_{\Omega} u \eta d x\right|_{\tau=0} ^{\tau=T} \\
& +\int_{Q_{T}}\left[u \eta_{t}-\sum_{i}\left(A_{i}(z)+b_{i}(z, u)\right) D_{i} \eta+d(z, u) \eta\right] d x d t y=0
\end{aligned}
$$

with an arbitrary $s \in \mathbb{N}$. It follows then that identity (3.22) holds for every $\eta \in \mathbf{W}\left(Q_{T}\right)$. It remains to identify the limit functions $A_{i}$.

Lemma 3.5. For almost all $z \in Q_{T}$

$$
A_{i}(z)=a_{i}(z, u)\left|D_{i} u\right|^{p_{i}(z)-2} D_{i} u, \quad i=1, \ldots, n .
$$

Proof: We rely on the monotonicity of the operator $\mathcal{M}(s)=|s|^{p-2} s$ : $\forall \xi, \eta \in \mathbb{R}^{n}$

$$
(\mathcal{M}(\xi)-\mathcal{M}(\eta))(\xi-\eta) \geq \begin{cases}2^{-p}|\xi-\eta|^{p} & \text { if } 2 \leq p<\infty \\ (p-1)|\xi-\eta|^{2}\left(|\xi|^{p}+|\eta|^{p}\right)^{\frac{p-2}{p}} & \text { if } 1<p<2\end{cases}
$$

According to (3.24), for every $\xi \in \mathbf{Z}_{m}$

$$
\int_{Q_{T}} a_{i}\left(z, u^{(m)}\right)\left(\left|D_{i} u^{(m)}\right|^{p_{i}-2} D_{i} u^{(m)}-\left|D_{i} \xi\right|^{p_{i}-2} D_{i} \xi\right) D_{i}\left(u^{(m)}-\xi\right) d x d t \geq 0 .
$$

Let $\xi \in \mathbf{Z}_{m}$. It follows from (3.5) with the test-function $\eta=u^{(m)}-\xi$

$$
\begin{gathered}
\int_{Q_{T}}\left\{u^{(m)} \eta_{t}-\sum_{i}\left[a_{i}\left(z, u^{(m)}\right)\left|D_{i} \xi\right|^{p_{i}-2} D_{i} \xi+b_{i}\left(z, u^{(m)}\right)\right] D_{i} \eta\right. \\
-\left.\int_{\Omega} u^{(m)} \eta d x\right|_{t=0} ^{t=T} \geq 0 .
\end{gathered}
$$

Gathering (3.22) with this inequality, integrating by parts the term $u^{(m)} \eta_{t}$, and then letting $m \rightarrow \infty$ we conclude, following [36, pp. 158-161], that

$\forall \xi \in \mathbf{W}\left(Q_{T}\right) \quad \sum_{i} \int_{Q_{T}}\left[A_{i}(z)-a_{i}(z, u)\left|D_{i} \xi\right|^{p_{i}-2} D_{i} \xi\right] D_{i}(u-\xi) d x d t \geq 0$. 
Choosing now $\xi=u \pm \epsilon \zeta$ with $\epsilon>0$, simplifying and then letting $\epsilon \rightarrow 0$, we have

$$
\forall \zeta \in \mathbf{W}\left(Q_{T}\right) \quad \sum_{i} \int_{Q_{T}}\left[A_{i}(z)-a_{i}(z, u)\left|D_{i} u\right|^{p_{i}-2} D_{i} u\right] D_{i} \zeta d x d t=0 .
$$

We have shown that under the conditions of Theorems 3.1 and 3.2 the solution $u \in \mathbf{W}\left(Q_{T}\right)$ satisfies the identity

$$
\forall \zeta \in \mathbf{W}\left(Q_{T}\right) \quad \int_{Q}\left[u_{t} \zeta+\sum_{i}\left(a_{i}\left|D_{i} u\right|^{p_{i}-2} D_{i} u+b_{i}\right) D_{i} \zeta+d \zeta\right] d z=0 .
$$

Applying Proposition 2.5 and integrating by parts in the first term of (3.25), we complete the proof of Theorems 3.1 and 3.2.

\section{4. $L^{\infty}$ estimates}

\subsection{Global estimates.}

Theorem 4.1. Let the conditions of Theorem 3.1 be fulfilled and, additionally,

(4.1) $\forall k \in \mathbb{N} \sup \left\{\left.|s|^{p_{i}^{\prime}(z)-1}|| \frac{\partial b_{i}(z, s)}{\partial s}\right|^{p_{i}^{\prime}(z)}: z \in Q_{T}, s \in[-k, k]\right\}$ $=B_{k}<\infty$,

and $\forall s \in \mathbb{R}, z \in Q_{T}$,

(a) $\quad|d(z, s)| \leq d_{0}|s|+h_{d}(z)$,

(b) $\quad\left|\frac{\partial b_{i}(z, s)}{\partial x_{i}}\right| \leq b_{0}|s|+h_{b}(z)$

with finite nonnegative constants $d_{0}, b_{0}$. If $\left\|u_{0}\right\|_{\infty, \Omega}<\infty$, then the weak solution of problem (1.1) is bounded and satisfies the estimate

(4.3) $\|u(\cdot, t)\|_{\infty, \Omega} \leq \mathrm{e}^{C_{0} t}\left\|u_{0}\right\|_{\infty, \Omega}$

$$
+\mathrm{e}^{C_{0} t} \int_{0}^{t} \mathrm{e}^{-C_{0} t}\left(\left\|h_{b}(\cdot, t)\right\|_{\infty, \Omega}+\left\|h_{d}(\cdot, t)\right\|_{\infty, \Omega}\right) d t
$$

with $C_{0}=b_{0}+d_{0}$.

Proof: Let us fix $k \in \mathbb{N}$ and consider the auxiliary problem

$$
\left\{\begin{array}{l}
u_{t}-\sum_{i} \frac{d}{d x_{i}}\left[a_{i}\left|D_{i} u\right|^{p_{i}-2} D_{i} u+b_{i}\right]+d_{k}(z, u)=0 \text { in } Q_{T}, \\
u=0 \text { on } \Gamma_{T}, u(x, 0)=u_{0}(x) \text { in } \Omega
\end{array}\right.
$$


with

$$
d_{k}(z, u) \equiv d(z, \min \{|u| ; k\} \operatorname{sign} u)
$$

Since for every finite $k$

$$
|d(z, \min \{|u| ; k\} \operatorname{sign} u)| \leq d_{0} k^{\lambda-1}+h_{d},
$$

it follows from Theorem 3.1 that problem (4.4) has a weak solution $u(z)$ in the sense of Definition 3.1. Let us introduce the function

$$
u_{k}=\min \{|u|, k\} \operatorname{sign} u \equiv \begin{cases}k & \text { if } u>k, \\ u & \text { if }|u| \leq k, \\ -k & \text { if } u<-k .\end{cases}
$$

The function $u_{k}^{2 m-1}$ with $m \in \mathbb{N}$ can be taken for the test-function in (3.25). Let in (3.25) $t_{2}=t+h, t_{1}=t$, with $t, t+h \in(0, T)$. Observe that $d_{k}(z, u)=d\left(z, u_{k}\right)$. Then

$$
\begin{aligned}
& \frac{1}{2 m} \int_{t}^{t+h} \frac{d}{d t}\left(\int_{\Omega} u_{k}^{2 m}(x, t) d x\right) d t \\
& \quad+\sum_{i} \int_{t}^{t+h} \int_{\Omega}\left((2 m-1) a_{i} u_{k}^{2(m-1)}\left|D_{i} u_{k}\right|^{p_{i}}+b_{i}(z, u) D_{i} u_{k}^{2 m-1}\right) d x d t \\
& \quad+\int_{t}^{t+h} \int_{\Omega} d\left(z, u_{k}\right) u_{k}^{2 m-1} d x d t=0 .
\end{aligned}
$$

Dividing the last equality by $h$ and letting $h \rightarrow 0$, we have that $\forall$ a.e. $t \in$ $(0, T)$

$$
\begin{aligned}
\frac{1}{2 m} \frac{d}{d t} & \int_{\Omega} u_{k}^{2 m}(x, t) d x \\
& +\sum_{i} \int_{\Omega}\left((2 m-1) a_{i} u_{k}^{2(m-1)}\left|D_{i} u_{k}\right|^{p_{i}}+b_{i}(z, u) D_{i} u_{k}^{2 m-1}\right) d x \\
& +\int_{\Omega} d\left(z, u_{k}\right) u_{k}^{2 m-1} d x=0 .
\end{aligned}
$$

Indeed: by Lebesgue's dominated convergence theorem for every $\phi \in$ $L^{1}(0, T)$ and a.e. $t \in(0, T)$ there exists $\lim _{h \rightarrow 0} \int_{t}^{t+h} \phi(\tau) d \tau=\phi(t)$. Let us write (4.5) in the form: $\forall$ a.e. $t \in(0, T)$

$$
\begin{aligned}
& \text { (4.6) } \frac{1}{2 m} \frac{d}{d t} \int_{\Omega} u_{k}^{2 m}(x, t) d x+(2 m-1) \sum_{i} \int_{\Omega} a_{i} u_{k}^{2(m-1)}\left|D_{i} u_{k}\right|^{p_{i}} d x \\
& =\sum_{i=1}^{n} J_{i}+I
\end{aligned}
$$


Parabolic Equation with Variable Nonlinearity 377

where

$$
\begin{aligned}
& J_{i}=\int_{\Omega} b_{i}(z, u) D_{i} u_{k}^{2 m-1} d x \equiv \int_{\Omega} b_{i}\left(z, u_{k}\right) D_{i} u_{k}^{2 m-1} d x \\
& I=-\int_{\Omega} d\left(z, u_{k}\right) u_{k}^{2 m-1} d x
\end{aligned}
$$

Integrating by parts, we find that

$$
\begin{aligned}
J_{i}= & -\int_{\Omega} \frac{\partial b_{i}\left(z, u_{k}\right)}{\partial u} u_{k}^{2 m-1} D_{i} u_{k} d x \\
& -\int_{\Omega} \frac{\partial b_{i}\left(z, u_{k}\right)}{\partial x_{i}} u_{k}^{2 m-1} d x=J_{i}^{(1)}+J_{i}^{(2)} .
\end{aligned}
$$

Applying Young's and Hölder's inequalities and plugging (4.1)-(4.2), we estimate $J_{i}^{(1)}, J_{i}^{(2)}$ and $I$ as follows:

$$
\begin{aligned}
\left|J_{i}^{(1)}\right| \leq & \epsilon a_{0}(2 m-1) \int_{\Omega}\left|u_{k}\right|^{2(m-1)}\left|D_{i} u_{k}\right|^{p_{i}} d x \\
& +\frac{C\left(p_{i}, \epsilon\right)}{(2 m-1)^{\frac{1}{p_{i}-1}}} \int_{\Omega}\left(\left|u_{k}\right|^{p_{i}^{\prime}-1}\left|\frac{\partial b_{i}\left(z, u_{k}\right)}{\partial u}\right|^{p_{i}^{\prime}}\right)\left|u_{k}\right|^{2 m-1} d x \\
\leq & \epsilon a_{0}(2 m-1) \int_{\Omega}\left|u_{k}\right|^{2(m-1)}\left|D_{i} u_{k}\right|^{p_{i}} d x \\
& +B_{k} \frac{C\left(p_{i}, \epsilon\right)|\Omega| \frac{1}{2 m}}{(2 m-1)^{\frac{1}{p_{i}-1}}}\left(\int_{\Omega}\left|u_{k}\right|^{2 m} d x\right)^{1-\frac{1}{2 m}}, \\
\left|J_{i}^{(2)}\right| \leq & \left|\int_{\Omega} \frac{\partial b_{i}\left(z, u_{k}\right)}{\partial x_{i}} u_{k}^{2 m-1} d x\right| \leq \int_{\Omega}\left(b_{0}\left|u_{k}\right|+h_{b}\right)|u|_{k}^{2 m-1} d x \\
\leq & C \int_{\Omega}\left(|u|_{k}^{2 m}+h_{b}^{2 m}\right) d x \\
\leq & b_{0} \int_{\Omega}\left|u_{k}\right|^{2 m} d x+\left(\int_{\Omega} h_{b}^{2 m} d x\right)^{\frac{1}{2 m}}\left(\int_{\Omega}\left|u_{k}\right|^{2 m} d x\right)^{\frac{2 m-1}{2 m}} \\
|I| \leq & \int_{\Omega}\left|d\left(z, u_{k}\right)\right|\left|u_{k}\right|^{2 m-1} d x \leq \int_{\Omega}\left(d_{0}\left|u_{k}\right|+h_{d}\right)\left|u_{k}\right|^{2 m-1} d x \\
\leq & d_{0} \int_{\Omega}\left|u_{k}\right|^{2 m}+\left(\int_{\Omega} h_{d}^{2 m} d x\right)^{\frac{1}{2 m}}\left(\int_{\Omega}\left|u_{k}\right|^{2 m} d x\right)^{\frac{2 m-1}{2 m}} .
\end{aligned}
$$

Let us introduce the function

$$
y_{k}(t)=\left\|u_{k}(\cdot, t)\right\|_{2 m, \Omega} .
$$


Choosing $\epsilon$ sufficiently small and substituting the above estimates into (4.6), we arrive at the inequality for the function $y_{k}(t)$ :

$$
\begin{aligned}
y_{k}^{2 m-1} \frac{d y_{k}}{d t}(t) \leq B_{k} \frac{\sum_{i} C\left(p_{i}, \epsilon\right)|\Omega| \frac{1}{2 m}}{(2 m-1)^{\frac{1}{p_{i}-1}}} y_{k}^{2 m-1}+\left(b_{0}+d_{0}\right) y_{k}^{2 m}(t) \\
\quad+y_{k}^{2 m-1}\left(\left\|h_{b}(\cdot, t)\right\|_{2 m, \Omega}+\left\|h_{d}(\cdot, t)\right\|_{2 m, \Omega}\right),
\end{aligned}
$$

or

$$
\begin{aligned}
\frac{d y_{k}}{d t}(t) \leq B_{k} \frac{\sum_{i} C\left(p_{i}, \epsilon\right)|\Omega|^{\frac{1}{2 m}}}{(2 m-1)^{\frac{1}{p_{i}-1}}}+ & \left(b_{0}+d_{0}\right) y_{k}(t) \\
& +\left(\left\|h_{b}(\cdot, t)\right\|_{2 m, \Omega}+\left\|h_{d}(\cdot, t)\right\|_{2 m, \Omega}\right) .
\end{aligned}
$$

Multiplying this inequality by $\mathrm{e}^{-C_{0} t}, C_{0}=\left(b_{0}+d_{0}\right)$, and integrating over the interval $(0, t)$ we arrive at the estimate

$$
\begin{aligned}
\mathrm{e}^{-C_{0} t}\left\|u_{k}(\cdot, t)\right\|_{2 m, \Omega} \leq & \left\|u_{0}\right\|_{2 m, \Omega}+t B_{k} \frac{\sum_{i} C\left(p_{i}, \epsilon\right)|\Omega|^{\frac{1}{2 m}}}{(2 m-1)^{\frac{1}{p_{i}-1}}} \\
& +\int_{0}^{t} \mathrm{e}^{-C_{0} \tau}\left(\left\|h_{b}(\cdot, \tau)\right\|_{2 m, \Omega}+\left\|h_{d}(\cdot, \tau)\right\|_{2 m, \Omega}\right) d \tau
\end{aligned}
$$

which yields, as $m \rightarrow \infty$,

(4.8)

$$
\begin{aligned}
& \forall k \in \mathbb{N} \\
& \begin{aligned}
\left\|u_{k}(\cdot, t)\right\|_{\infty, \Omega} \leq & \mathrm{e}^{C_{0} t}\left\|u_{0}\right\|_{\infty, \Omega} \\
& +\mathrm{e}^{C_{0} t} \int_{0}^{t} \mathrm{e}^{-C_{0} \tau}\left(\left\|h_{b}(\cdot, \tau)\right\|_{\infty, \Omega}+\left\|h_{d}(\cdot, \tau)\right\|_{\infty, \Omega}\right) d \tau \\
\equiv & K .
\end{aligned}
\end{aligned}
$$

The right-hand side of this estimate does not depend on $k$. Let us choose now $k \geq K+1$. Under this choice of $k$

$$
u_{k} \equiv \min \{|u| ; k\} \operatorname{sign} u=u, \quad d\left(z, u_{k}\right) \equiv d_{k}(z, u) \equiv d(z, u),
$$

which means that the solution of problem (4.4) with $k \geq K+1$ is, in fact, a solution of problem (1.1) which satisfies estimate (4.3).

Remark 4.1. It is worth mentioning here paper [44] which addresses the question of local boundedness of solutions to equation (1.1) with anisotropic but constant growth conditions. The method of proof is based on application of suitable embedding theorems in the anisotropic Sobolev spaces. 
4.2. Global existence via boundedness. Let us consider the case when in equation (1.1) the term $d(z, u)$ is of the special form:

$$
d(z, u)=d_{1}(z, u)|u|^{\sigma(z)-2} u+d_{2}(z, u)|u|^{\lambda-2} u+h_{d}(z),
$$

with

$$
\begin{gathered}
0<d_{01} \leq d_{1}(z, u)<\infty, \quad\left|d_{2}(z, u)\right| \leq d_{02}<\infty, \\
d_{01}, d_{02}, \lambda=\text { const }>0 .
\end{gathered}
$$

If $\sigma(z)$ and $\lambda$ satisfy conditions (1.3), (1.4), the existence of a weak solution follows from Theorems 3.1 and 3.2. If we additionally assume that the conditions of Theorem 4.1 are fulfilled, then this weak solution is bounded. We now turn to the study of the case

$$
2<\lambda<\sigma^{-} \leq \sigma(z) \leq \sigma^{+}<\infty,
$$

which does not fall into the scope of Theorems 3.1, 3.2, and 4.1. Let us take a positive number $R_{0}<\infty$ such that $\forall z \in Q_{T}$

(4.12) $\mathcal{P}\left(z, R_{0}\right) \equiv d_{01} R_{0}^{\sigma(z)-1}-d_{02} R_{0}^{\lambda-1}-b_{0} R_{0}-\sup _{Q_{T}}\left|h_{d}(z)\right|-\sup _{Q_{T}}\left|h_{b}(z)\right| \geq 0$.

Because of condition $\sigma^{-}>\lambda>2$, such a number always exists, provided that

$$
\sup _{Q_{T}}\left|h_{b}\right|+\sup _{Q_{T}}\left|h_{d}\right|<\infty .
$$

Theorem 4.2. Let the coefficients $a_{i}, b_{i}$ and the exponents $p_{i}$, satisfy the conditions of Theorem 4.1, and let $d(z, u)$ satisfy condition (4.9). Let us assume that $\sigma(z)$ is measurable in $Q_{T}$ and that conditions (4.9)-(4.12) are fulfilled. Then problem (1.1) has in $Q_{T}$ at least one bounded weak solution satisfying the estimate

$$
\|u\|_{\infty, Q_{T}} \leq \max \left\{\sup _{\Omega}\left|u_{0}\right| ; R_{0}\right\} .
$$

Remark 4.2. The conditions of Theorem 4.2 are surely fulfilled for the diffusion-absorption equation

$$
u_{t}=\Delta_{p(z)} u-|u|^{\sigma(z)-2} u+h_{d}(z), \quad \sigma(z)>1 .
$$

In this case $\|u\|_{\infty, Q_{T}} \leq \sup _{\Omega}\left|u_{0}\right|+\left\|h_{d}\right\|_{\infty, Q_{T}}$.

Proof of Theorem 4.2: Fix an arbitrary finite number $R>0$ and consider the regularized problem

$$
\left\{\begin{array}{l}
u_{t}-\sum_{i} \frac{d}{d x_{i}}\left[a_{i}\left|D_{i} u\right|^{p_{i}(z)-2} D_{i} u+b_{i R}(z, u)\right]+d_{R}(z, u)=0 \text { in } Q_{T}, \\
u=0 \text { on } \Gamma_{T}, u(x, 0)=u_{0}(x) \text { in } \Omega
\end{array}\right.
$$


with

$$
\begin{aligned}
d_{R}(z, u) & =d_{1}(z, u)\left|u_{R}\right|^{\sigma(z)-2} u_{R}+d_{2}\left(z, u_{R}\right)+h_{d}(z), \\
b_{i R}(z, u) & =b_{i}\left(z, u_{R}\right),
\end{aligned}
$$

and

$$
u_{R}=\min \{|u|, R\} \operatorname{sign} u, \quad D_{i} u_{R} \equiv \begin{cases}0 & \text { if }|u|>R, \\ D_{i} u & \text { if }|u| \leq R .\end{cases}
$$

The regularized problem (4.14) has a global weak solution. Moreover, since $b_{i}$ satisfy the conditions of Theorem 4.1, this solution is globally in time bounded: $\|u\|_{\infty, Q_{T}} \leq C(R)$. The theorem will be proved if we show that the constant $C(R)$ is in fact independent of $R$. Let us set

$$
R=\max \left\{R_{0}, \sup _{\Omega}\left|u_{0}\right|\right\}
$$

with $R_{0}$ satisfying the inequality $\mathcal{P}\left(z, R_{0}\right) \geq 0$. Let us take for the test-function in (3.25) the function

$$
u_{+}=\max \{u-R, 0\}, \quad D_{i} u_{+} \equiv \begin{cases}D_{i} u & \text { if } u>R, \\ 0 & \text { if } u \leq R .\end{cases}
$$

Arguing like in the proof of Theorem 4.1 we arrive at the equality

$$
\begin{aligned}
\frac{1}{2} \frac{d}{d t} \int_{\Omega} u_{+}^{2}(x, t) d x & +\sum_{i} \int_{\Omega}\left(a_{i}\left|D_{i} u_{+}\right|^{p_{i}}+b_{i}\left(z, u_{R}\right) D_{i} u_{+}\right) d x \\
& +\int_{\Omega} d_{R}(z, u) u_{+} d x=0 \quad \forall \text { a.e. } t \in(0, T),
\end{aligned}
$$

which can be written in the form

$$
\begin{aligned}
& \forall \text { a.e. } t \in(0, T) \\
& \frac{1}{2} \frac{d}{d t} \int_{\Omega} u_{+}^{2} d x+\sum_{i} \int_{\Omega} a_{i}\left|D_{i} u_{+}\right|^{p_{i}} d x+I \equiv \sum_{i=1}^{n}\left(J_{i}^{(1)}+J_{i}^{(2)}\right) .
\end{aligned}
$$

In the last relation

$$
\begin{aligned}
& I=\int_{\Omega}( d_{1}(z, u)(\min \{|u|, R\})^{\sigma(z)-1} \operatorname{sign} u \\
&\left.+d_{2}\left(z,(\min \{|u|, R\})^{\lambda-1} \operatorname{sign} u\right)+h_{d}(z)\right) u_{+} d x, \\
& J_{i}^{(1)}=-\int_{\Omega} \frac{\partial b_{i}\left(z, u_{R}\right)}{\partial u} u_{+} D_{i} u_{R} d x=0, \quad J_{i}^{(2)}=\int_{\Omega} \frac{\partial b_{i}\left(z, u_{R}\right)}{\partial x_{i}} u_{+} d x .
\end{aligned}
$$


The terms $J_{i}^{(j)}$ are estimated exactly like in the proof of Theorem 4.1:

$$
\left|J_{i}^{(2)}\right| \leq\left|\int_{\Omega} \frac{\partial b_{i}\left(z, u_{R}\right)}{\partial x_{i}} u_{+} d x\right| \leq \int_{\Omega}\left(b_{0} R+\left|h_{b}\right|\right) u_{+} d x .
$$

Further,

$$
I \geq \int_{\Omega}\left(d_{01} R^{\sigma(z)-1}-d_{02} R^{\lambda-1}-\sup _{Q_{T}}\left|h_{d}\right|\right) u_{+} d x .
$$

Gathering these estimates we find that

$$
\frac{1}{2} \frac{d}{d t} \int_{\Omega} u_{+}^{2} d x+\sum_{i} \int_{\Omega} a_{i}\left|D_{i} u_{+}\right|^{p_{i}} d x+\int_{\Omega} \mathcal{P}\left(z, R_{0}\right) u_{+} \leq 0 .
$$

Since $\mathcal{P}\left(z, R_{0}\right) \geq 0$ by the choice of $R_{0}$, and $u_{+}(x, 0)=0$ by the choice of $R$, the last inequality yields

$$
\forall \text { a.e. } z \in Q \quad u_{+}(z)=0,
$$

whence $u(z) \leq R$ a.e. in $Q_{T}$. The same argument shows that

$$
u_{-}(z)=\max \{-u(z)-R, 0\}=0
$$

and, finally,

$$
|u(z)| \leq R=\max \left\{\sup _{\Omega}\left|u_{0}(x)\right|, R_{0}\right\} .
$$

This inequality means that

$$
b_{i R}(z, u) \equiv b_{i}(z, u), \quad d_{R}(z, u) \equiv d(z, u),
$$

which completes the proof.

4.3. Local existence via boundedness. Let us consider problem (1.1) with the term $d(z, u)$ satisfying the growth condition

$$
|d(z, u)| \leq d_{0}|u|^{\lambda-1}+h_{d}(z), \quad \lambda=\text { const }>2 .
$$

For $0 \leq \lambda \leq 2$ the existence of a global bounded solution to problem (1.1) is proved in Theorem 3.1. The next theorem asserts the existence of local bounded solution in the case $\lambda>2$. 
Theorem 4.3. Let us assume that in the conditions of Theorems 3.1 and 4.1 the growth condition on the function $d(z, u)$ is substituted by (4.17). Then for every $u_{0} \in L^{\infty}(\Omega)$ there exists $\theta \in(0, T]$ depending on

$$
\lambda, b_{0}, d_{0},\left\|u_{0}\right\|_{L^{\infty}(\Omega)},\left\|h_{d}\right\|_{L^{1}\left(0, \theta ; L^{\infty}(\Omega)\right)} \text { and }\left\|h_{b}\right\|_{L^{1}\left(0, \theta ; L^{\infty}(\Omega)\right)}
$$

such that in the cylinder $Q_{\theta}$ problem (1.1) has at least one weak solution $u \in \mathbf{W}\left(Q_{\theta}\right)$ such that $u_{t} \in \mathbf{W}^{\prime}\left(Q_{\theta}\right)$ and $\|u\|_{\infty, Q_{\theta}}<\infty$. The solution can be continued to the interval $\left[0, T^{*}\right]$, where

$$
T^{*}=\sup \left\{\theta \in[0, T]:\|u\|_{\infty, Q_{\theta}}<\infty\right\} .
$$

Proof: Let us consider the auxiliary problem

$$
\left\{\begin{array}{l}
u_{t}-\sum_{i} D_{i}\left(a_{i}\left|D_{i} u\right|^{p_{i}(z)-2} D_{i} u+b_{i}\right)+d_{r}(z, u)=0 \quad \text { in } Q_{T} \\
u=0 \text { on } \Gamma, u(x, 0)=u_{0}(x) \text { in } \Omega
\end{array}\right.
$$

with the right-hand side

$$
d_{r}(z, u)=d(z, \min \{|u|, r\} \operatorname{sign} u), \quad r=\text { const }>1 .
$$

As in the proof of Theorem 4.1, we will make use of the fact that

$$
\left|d_{r}(z, u)\right| \leq d_{0} r^{\lambda-1}+h_{d}(z), \quad d_{r}(z, u)=d(z, u) \quad \text { if } r \geq u .
$$

By Theorems 3.1 and 4.1, for every $r>1$ the regularized problem (4.18) has a global bounded weak solution $u(z)$. Let us show that the function $w(t)=\|u(\cdot, t)\|_{\infty, \Omega}$ can be estimated by a constant which does not depend on $r$. Following the proof of Theorem 4.1 we find that the solution of (4.18) satisfies inequality (4.3) with $C_{0}=b_{0}$ and $h_{d}$ substituted by $h_{d}+d_{0} r^{\lambda-1}$ :

$$
\begin{aligned}
\|u(\cdot, t)\|_{\infty, \Omega} \leq & \mathrm{e}^{b_{0} t}\left\|u_{0}\right\|_{\infty, \Omega}+\mathrm{e}^{b_{0} t} \int_{0}^{t} \mathrm{e}^{-b_{0} t}\left\|h_{b}(\cdot, t)\right\|_{\infty, \Omega} d t \\
& +\mathrm{e}^{b_{0} t} \int_{0}^{t} \mathrm{e}^{-b_{0} t}\left\|h_{d}(\cdot, t)\right\|_{\infty, \Omega} d t+d_{0} r^{\lambda-1} t \mathrm{e}^{b_{0} t} \equiv \mathcal{R}(r, t) .
\end{aligned}
$$

For every fixed $r>1$

$$
\mathcal{R}(r, t) \rightarrow\left\|u_{0}\right\|_{\infty, \Omega} \quad \text { as } t \rightarrow 0,
$$

whence for every $r \geq\left\|u_{0}\right\|_{\infty, \Omega}$ there is $t \equiv t(r)$ such that

$$
\forall t \in[0, t(r)] \quad\|u(\cdot, t)\|_{\infty, \Omega} \leq r .
$$

It follows that for $r$ and $t(r)$ chosen in this way $\|u(\cdot, t)\|_{\infty, \Omega} \leq r$ for all $t \leq$ $t(r)$, i.e., the constructed solution of the regularized problem (4.18) is a weak solution of problem (1.1) in the cylinder $Q_{t(r)}$. The possibility of continuation of this solution to the maximal interval $\left[0, T^{*}\right]$ follows from 
the fact that the function $u(x, t(r))$ possesses the same properties as the initial function $u_{0}$.

\section{Uniqueness theorems}

In this section we study the question of uniqueness of weak solutions to the problem

$$
\left\{\begin{array}{l}
u_{t}-\sum_{i} \frac{d}{d x_{i}}\left[a_{i}(z, u)\left|D_{i} u\right|^{p_{i}(z)-2} D_{i} u\right]+d(z, u)=0 \quad \text { in } Q \\
u=0 \text { on } \Gamma, u(x, 0)=u_{0}(x) \text { in } \Omega
\end{array}\right.
$$

The weak solution is understood in the sense of Definition 3.1.

Let us assume that the functions $a_{i}$ are continuous with the module of continuity $\omega$,

$$
\left|a_{i}\left(z, u_{1}\right)-a\left(z, u_{2}\right)\right| \leq \omega\left(\left|u_{1}-u_{2}\right|\right),
$$

and claim that the function $\omega$ is nonnegative and satisfies the condition

$$
\int_{\epsilon}^{1} \frac{\mathrm{d} s}{\omega^{\alpha}(s)} \rightarrow \infty \quad \text { as } \epsilon \rightarrow 0+\quad \text { for some } 1<\alpha<\frac{p^{+}}{p^{+}-1} .
$$

Without loss of generality we may assume that $p^{+} \geq 2$.

Theorem 5.1. Let

$1<p^{-} \leq p_{i}(z) \leq p^{+}<\infty, \quad 0<a_{0} \leq a_{i}(z, u) \leq a_{1}<\infty, \quad i=1, \ldots, n$.

Problem (5.1) does not admit more than one solution $u \in \mathbf{W}\left(Q_{T}\right)$ if conditions (5.2), (5.3) are fulfilled and

$$
u \mapsto d(z, u) \text { is a nondecreasing function. }
$$

Proof: We argue by contradiction. Let us assume that problem (5.1) admits two different solutions $u_{1}, u_{2} \in \mathbf{W}\left(Q_{T}\right)$ and there is $\delta>0$ such that for some $\tau \in(0, T] w=u_{2}-u_{1}>\delta$ on the set

$$
\Omega_{\delta}=\Omega \cap\{x: w(x, t)>\delta\} \quad \text { and } \quad\left|\Omega_{\delta}\right|=\mu>0 .
$$

We will show that this assumption leads to a contradiction unless $\mu=0$. Not loosing generality we assume that $t=T$. Set

$$
d_{i} \equiv d\left(z, u_{i}\right), \quad a_{i j} \equiv a_{j}\left(z, u_{i}\right), \quad i=1,2, j=1, \ldots, n .
$$


By the definition of weak solution, for every test-function $\zeta \in \mathbf{Z}$ and $\tau \in[0, T]$

$$
\begin{gathered}
\int_{Q_{\tau}}\left(w_{t} \zeta+\sum_{i=1}^{n} a_{2 i}\left(\left|D_{i} u_{2}\right|^{p_{i}-2} D_{i} u_{2}-\left|D_{i} u_{1}\right|^{p_{i}-2} D_{i} u_{1}\right) D_{i} \zeta+\left(d_{2}-d_{1}\right) \zeta\right) d z \\
\left.+\int_{Q_{\tau}} \sum_{i=1}^{n}\left(a_{2 i}-a_{1 i}\right)\left|D_{i} u_{1}\right|^{p_{i}-2} D_{i} u_{1}\right) D_{i} \zeta d z=0 .
\end{gathered}
$$

Let us denote

$$
\begin{aligned}
A\left(u_{2}, u_{1}\right) & =d_{2}-d_{1} \\
J\left(u_{2}, u_{1}, \zeta\right) & =-\int_{Q_{\tau}} \sum_{i=1}^{n}\left(a_{2 i}-a_{1 i}\right)\left|D_{i} u_{1}\right|^{p_{i}-2} D_{i} u_{1} D_{i} \zeta d z
\end{aligned}
$$

and write (5.5) in the form

$$
\begin{array}{r}
\int_{Q_{\tau}}\left(w_{t} \zeta+\sum_{i=1}^{n} a_{2 i}\left(\left|D_{i} u_{2}\right|^{p_{i}-2} D_{i} u_{2}-\left|D_{i} u_{1}\right|^{p_{i}-2} D_{i} u_{1}\right) D_{i} \zeta\right. \\
\left.+A\left(u_{2}, u_{1}\right) \zeta\right) d z \\
=J\left(u_{2}, u_{1}, \zeta\right) .
\end{array}
$$

Let us introduce the functions

$$
F_{\epsilon}(\xi)=\left\{\begin{array}{ll}
\int_{\epsilon}^{\xi} \frac{\mathrm{d} s}{\omega^{\alpha}(s)} & \xi>\epsilon, \\
0 & \xi \leq \epsilon,
\end{array} \quad G_{\epsilon}(\eta)= \begin{cases}\int_{\epsilon}^{\eta} F_{\epsilon}(s) d s & \eta>\epsilon \\
0 & \eta \leq \epsilon\end{cases}\right.
$$

depending on the parameters $\delta \geq \epsilon>0$, and with the function $\omega(\cdot)$ defined in (5.3). The definition of $F_{\epsilon}$ and (5.4) yield:

$$
\forall u, v \in \mathbb{R} \quad A(u, v) F_{\epsilon}(u-v) \geq 0 .
$$

Set $Q_{\epsilon, \tau} \equiv\left\{z \in Q_{\tau}: w>\epsilon\right\}$. By the definition of $F_{\epsilon}$

$$
D_{i} F_{\epsilon}(w)= \begin{cases}\frac{D_{i} w}{\omega^{\alpha}(w)} & \text { in } Q_{\epsilon}, \\ 0 & \text { in } Q \backslash Q_{\epsilon} .\end{cases}
$$


Letting in $(5.6) \zeta=F_{\epsilon}(w)$, we obtain:

$$
\begin{aligned}
& \int_{\Omega_{\epsilon}} G_{\epsilon}(w(x, \tau)) d x \\
& +\int_{Q_{\epsilon, \tau}}\left(\sum_{i=1}^{n} a_{2 i}\left(\left|D_{i} u_{2}\right|^{p_{i}-2} D_{i} u_{2}-\left|D_{i} u_{1}\right|^{p_{i}-2} D_{i} u_{1}\right) \frac{D_{i} w}{\omega^{\alpha}(w)}\right. \\
& \left.+A\left(u_{2}, u_{1}\right) F_{\epsilon}(w)\right) d z \\
& \equiv J\left(u_{2}, u_{1}, F_{\epsilon}(w)\right) .
\end{aligned}
$$

Notice that since $\delta \geq \epsilon$, then $\Omega_{\delta} \subseteq \Omega_{\epsilon},\left|\Omega_{\epsilon}\right| \geq\left|\Omega_{\delta}\right|>\mu$ and, by virtue of (5.3),

$$
\int_{\Omega_{\epsilon}} G_{\epsilon}(w(x, \tau)) d x \geq \mu F_{\epsilon}(\delta) \rightarrow \infty \quad \text { as } \epsilon \rightarrow 0+.
$$

Let us consider first the case $p_{i} \geq 2$. By virtue of (1.3) and the first inequality of (3.24)

$$
a_{0} \frac{\left|D_{i} w\right|^{p_{i}}}{\omega^{\alpha}(w)} \leq a_{2 i}\left(\left|D_{i} u_{1}\right|^{p_{i}-2} D_{i} u_{1}-\left|D_{i} u_{2}\right|^{p_{i}-2} D_{i} u_{2}\right) \frac{D_{i} w}{\omega^{\alpha}(w)} .
$$

According to (5.3)

$$
\frac{p_{i}}{p_{i}-1} \geq \frac{p^{+}}{p^{+}-1} \geq \alpha>1 .
$$

Applying Young's inequality, we may estimate the integrand of $J$ in the following way:

$$
\begin{aligned}
\left.\left|\left(a_{21 i}-a_{1 i}\right)\right| D_{i} u_{1}\right|^{p_{i}-2} D_{i} u_{1} \frac{D_{i} w}{\omega^{\alpha}(w)} \mid \\
\leq \omega(w)\left|D_{i} u_{1}\right|^{p_{i}-1} \frac{\left|D_{i} w\right|}{\omega^{\alpha}(w)} \\
\leq \frac{a_{0}}{2} \frac{\left|D_{i} w\right|^{p_{i}}}{\omega^{\alpha}(w)}+C\left(a_{0}, p^{+}\right)\left|D_{i} u_{1}\right|^{p_{i}} \omega^{p^{\prime}-\alpha}(w) \\
\leq \frac{a_{0}}{2} \frac{\left|D_{i} w\right|^{p_{i}}}{\omega^{\alpha}(w)}+C\left(a_{0}, p^{+}\right)\left|D_{i} u_{1}\right|^{p_{i}} .
\end{aligned}
$$


Let now $1<p^{-} \leq p_{i}<2$. Applying (1.3) and the second inequality of (3.24) we have

$$
\begin{aligned}
& a_{0}\left(p^{-}-1\right)\left(\left|D_{i} u_{1}\right|+\left|D_{i} u_{2}\right|\right)^{p_{i}-2} \frac{\left|D_{i} w\right|^{2}}{\omega^{\alpha}(w)} \\
& \quad \leq a_{2 i}\left(\left|D_{i} u_{2}\right|^{p_{i}-2} D_{i} u_{2}-\left|D_{i} u_{1}\right|^{p_{i}-2} D_{i} u_{1}\right) \frac{D_{i} w}{\omega^{\alpha}(w)}
\end{aligned}
$$

and

$$
\begin{aligned}
&\left.\left|\left(a_{2 i}-a_{1 i}\right)\right| D_{i} u_{1}\right|^{p_{i}-2} D_{i} u_{1} \frac{D_{i} w}{\omega^{\alpha}(w)} \mid \\
& \leq\left|\omega(w)\left(\left|D_{i} u_{1}\right|+\left|D_{i} u_{2}\right|\right)^{p_{i}-1} \frac{D_{i} w}{\omega^{\alpha}(w)}\right| \\
& \leq\left|\omega(w)\left(\left|D_{i} u_{1}\right|+\left|D_{i} u_{2}\right|\right)^{p_{i}-1} \frac{D_{i} w}{\omega^{\alpha}(w)}\right| \\
& \leq \frac{a_{0}\left(p^{-}-1\right)}{2}\left(\left|D_{i} u_{1}\right|+\left|D_{i} u_{2}\right|\right)^{p_{i}-2} \frac{\left|D_{i} w\right|^{2}}{\omega^{\alpha}(w)} \\
&+C \omega^{2-\alpha}(w)\left(\left|D_{i} u_{2}\right|+\left|D_{i} u_{1}\right|\right)^{p_{i}} \\
& \leq \frac{a_{0}\left(p^{-}-1\right)}{2}\left(\left|D_{i} u_{1}\right|+\left|D_{i} u_{2}\right|\right)^{p_{i}-2} \frac{\left|D_{i} w\right|^{2}}{\omega^{\alpha}(w)} \\
&+\widetilde{C}\left(\left|D_{i} u_{2}\right|+\left|D_{i} u_{1}\right|\right)^{p_{i}}
\end{aligned}
$$

with

$$
1<\alpha \leq \frac{p^{+}}{p^{+}-1} \leq 2
$$

Plugging the pointwise estimates (5.11), (5.12) and (5.13), (5.14) into (5.9) and dropping the nonnegative terms, we arrive at the inequality

$$
\int_{\Omega_{\epsilon}} G_{\epsilon}(w(x, \tau)) d x \leq \widetilde{C} \int_{Q_{\epsilon, \tau}} \sum_{i=1}^{n}\left(\left|D_{i} u_{1}\right|+\left|D_{i} u_{2}\right|\right)^{p_{i}} d z=\widehat{C}
$$

with a constant $\widetilde{C}$ independent of $\epsilon$. Gathering this inequality with (5.10) we obtain the needed contradiction. This means that $\mu=0$ and $w \leq 0$ a.e. in $Q_{T}$. To complete the proof it suffices to replace $u_{1}$ and $u_{2}$. 
The above arguments can be extended to non-monotone functions $d(z, u)$. Let

$$
u_{t}-\sum_{i=1}^{n} \frac{d}{d x_{i}}\left[a_{i}(z, u)\left(\left|D_{i} u_{1}\right|^{p_{i}-2} D_{i} u\right]+d(z, u)=0\right.
$$

We assume that

$$
\left\{\begin{array}{l}
\left|a_{i}\left(z, u_{1}\right)-a_{i}\left(z, u_{2}\right)\right| \leq C_{1} \omega\left(\left|u_{1}-u_{2}\right|\right) \\
\omega^{\alpha}(s)=s^{2} \\
\left|d\left(z, u_{1}\right)-d_{i}\left(z, u_{2}\right)\right| \leq C_{2}\left|u_{1}-u_{2}\right|
\end{array}\right.
$$

It is easy to calculate that in this case

$$
F_{\epsilon}(s)=\left\{\begin{array}{ll}
\frac{1}{\epsilon}-\frac{1}{s} & \text { for } s>\epsilon, \\
0 & \text { otherwise, }
\end{array} \quad G_{\epsilon}(s)= \begin{cases}\frac{s}{\epsilon}-1-\ln \left(\frac{s}{\epsilon}\right) & \text { for } s>\epsilon \\
0 & \text { otherwise }\end{cases}\right.
$$

Proposition 5.1. There exists a positive number $\mu>2$ such that

$$
s F_{\epsilon}(s) \leq \begin{cases}2 G_{\epsilon}(s) & \text { for } s \geq \mu \epsilon \\ \text { const } & \text { for } \epsilon \leq s \leq \mu \epsilon .\end{cases}
$$

Proof: Set $z=s / \epsilon$ and introduce the function

$$
f(z)=2 G_{\epsilon}(s)-s F_{\epsilon}(s) \equiv z-1-2 \ln z .
$$

Obviously,

$$
\begin{aligned}
& f(1)=0 \\
& f(z) \rightarrow \infty \text { as } z \rightarrow \infty \\
& f^{\prime}(z)=1-\frac{2}{z} \geq 0 \\
& f^{\prime \prime}(z)=\frac{2}{z^{2}} \geq 0 \text { if } z \geq 2 .
\end{aligned}
$$

Since $f(z)$ is monotone increasing for $z>2$ and tends to infinity as $z \rightarrow$ $\infty$, there is $\mu \geq 2$ such that $f(z) \geq 0$ for $z \geq \mu$. For $z \in[1, \mu]$

$$
s F_{\epsilon}(s)=z-1 \leq \mu-1 \text {. }
$$

Theorem 5.2. Let in the conditions of Theorem 5.1 condition (5.4) is substituted by condition (5.17). Then the weak solution of problem (5.1) is unique. 
Proof: We will adapt the proof of Theorem 5.1. Let $u_{1}, u_{2}$ be two different solutions of problem (1.1). Set $u=u_{1}-u_{2}$. Following the proof of Theorem 5.1 we arrive at the relation

$$
\begin{aligned}
& \int_{\Omega_{\epsilon}} G_{\epsilon}(u(x, \tau)) d x \\
& \quad+\int_{Q_{\epsilon, \tau}}\left(\sum_{i=1}^{n} a_{1 i}\left(\left|D_{i} u_{1}\right|^{p_{i}-2} D_{i} u_{1}-\left|D_{i} u_{2}\right|^{p_{i}-2} D_{i} u_{2}\right) \frac{D_{i} u}{u^{2}}\right) d z \\
& =I_{1}+I_{2}
\end{aligned}
$$

with

$$
\begin{aligned}
& I_{1}=-\int_{Q \epsilon} \sum_{i=1}^{n}\left(a_{1 i}-a_{2 i}\right)\left|D_{i} u_{2}\right|^{p_{i}-2} D_{i} u_{2} \frac{D_{i} u}{u^{2}} d z, \\
& I_{2}=-\int_{Q \epsilon}\left(d\left(z, u_{1}\right)-d\left(z, u_{2}\right)\right) F_{\epsilon}(u) d z .
\end{aligned}
$$

The difference between this case and the one studied in Theorem 5.1 is that now the term $I_{2}$ is not sign-defined. By Proposition 5.1

$$
\begin{aligned}
\left|I_{2}\right| & \leq C \int_{Q \epsilon} u F_{\epsilon}(u) d z \\
& =C \int_{0}^{t}\left(\int_{\Omega \cap(\epsilon \leq u \leq \mu \epsilon)} \cdots+\int_{\Omega \cap(\mu \epsilon \leq u)} \cdots\right) d t \equiv I_{21}+I_{22},
\end{aligned}
$$

whence

$$
I_{21} \leq C \int_{0}^{t}\left(\int_{\Omega \cap(\epsilon \leq u \leq \mu \epsilon)} G_{\epsilon}(u) d x\right) d t \leq C \int_{0}^{t}\left(\int_{\Omega_{\epsilon}} G_{\epsilon}(u) d x\right) d t,
$$

$I_{22} \leq C|\Omega| T$.

Let us introduce the function

$$
Y(t)=\int_{\Omega \epsilon} G_{\epsilon}(u) d x
$$

Substituting the above inequalities into (5.19) and taking into account (3.24), we find that the function $Y(t)$ satisfies the Gronwall type inequality

$$
Y(t) \leq C\left(\int_{0}^{t} Y(s) d s+\sum_{i=1}^{n} \int_{Q \epsilon}\left(\left|D_{i} u_{1}\right|^{p_{i}}+\left|D_{i} u_{2}\right|^{p_{i}}\right) d z+1\right) .
$$

It follows that $Y(t) \leq K$, which contradicts condition (5.10). 
Corollary 5.1 (Comparison principle). Let $u, v \in \mathbf{W}\left(Q_{T}\right)$ be two weak solutions of problem (5.1) such that $u(x, 0) \leq v(x, 0)$ a.e. in $\Omega$. If the coefficients and the nonlinearity exponents satisfy the conditions of Theorem 5.1 or Theorem 5.2, then $u \leq v$ a.e. in $Q_{T}$.

\section{Regularity of solutions for a class of model equations}

Let us consider the following simplified version of problem (1.1):

(6.1) $\left\{\begin{array}{l}u_{t}-\sum_{i=1}^{n} \frac{d}{d x_{i}}\left(a_{i}(z)\left|D_{i} u\right|^{p_{i}(z)-2} D_{i} u\right)+c(z)|u|^{\sigma(z)-2} u=f(z) \text { in } Q_{T}, \\ u=0 \text { on } \Gamma, u(x, 0)=u_{0}(x) \text { in } \Omega .\end{array}\right.$

We want to trace the dependence of the regularity of weak solutions on the regularity of the data, especially, on the properties of the exponents $p_{i}(z)$ and $\sigma(z)$. Let us accept the notations

$$
\begin{aligned}
\lambda_{p}(t) & =\sum_{i=1}^{n} \max _{\bar{\Omega}}\left|p_{i t}(x, t)\right|, & \lambda_{a}(t) & =\sum_{i=1}^{n} \max _{\bar{\Omega}}\left|a_{i t}(x, t)\right|, \\
\lambda_{\sigma}(t) & =\max _{\bar{\Omega}}\left|\sigma_{t}(x, t)\right|, & \lambda_{c}(t) & =\max _{\bar{\Omega}}\left|c_{t}(x, t)\right| .
\end{aligned}
$$

Theorem 6.1. Let us assume that

a) $a_{i}, p_{i}$ satisfy the conditions of Theorem 3.1,

b) $p_{i t}(z) \leq 0$ for a.a. $z \in Q_{T}$,

c) $\sigma(z)$ and $c(z)$ are bounded measurable in $Q_{T}$ functions, $\sigma_{t}(z)$ exists a.e. in $Q_{T}$, and

$$
\sigma_{t}(z) \leq 0, \quad 0 \leq c_{0} \leq c(z) \quad \text { a.e. in } Q_{T},
$$

d) $\lambda_{p}(t), \lambda_{\sigma}(t), \lambda_{a}(t), \lambda_{c}(t) \in L^{1}(0, T)$ and

$$
\int_{0}^{T}\left(\lambda_{p}(t)+\lambda_{a}(t)+\lambda_{\sigma}(t)+\lambda_{c}(t)\right) d t=K<\infty,
$$

e) $u_{0} \in L^{\sigma(\cdot, 0)}(\Omega), D_{i} u_{0} \in L^{p_{i}(\cdot, 0)}(\Omega)$. 
Then the weak solution of problem (6.1) satisfies the estimate

$$
\begin{aligned}
\sup _{t \in(0, T)} & \int_{\Omega}\left[\sum_{i=1}^{n} a_{i}\left|D_{i} u\right|^{p_{i}}+c|u|^{\sigma}\right] d x+\int_{Q_{T}}\left|u_{t}\right|^{2} d z \\
& +\int_{Q_{T}}\left(\sum_{i=1}^{n} a_{i}\left|D_{i} u\right|^{p_{i}}|\ln | D_{i} u||\left|p_{i t}\right|+c|u|^{\sigma}|\ln | u||\left|\sigma_{t}\right|\right) d z \\
\leq & C\left(\int_{\Omega}\left(\sum_{i=1}^{n}\left|D_{i} u_{0}\right|^{p_{i}(x, 0)}+\left|u_{0}\right|^{\sigma(x, 0)}\right) d x+1\right)
\end{aligned}
$$

with an absolute constant $C=C\left(p^{ \pm}, \sigma^{ \pm}, q, T, K\right)$.

Proof: Let us recall that under the conditions of Theorem 6.1 a weak solution of problem (6.1) can be obtained as the limit of the sequence of Galerkin's approximations (see the proof of Theorem 3.1)

$$
u^{(m)}=\sum_{k=1}^{m} u_{k}^{(m)}(t) \psi_{k}(x), \quad \psi_{k}(x) \in W_{0}^{1, p^{+}}(\Omega), \quad p^{+}=\max _{i} \sup _{Q_{T}} p_{i}(z),
$$

where the system $\left\{\psi_{k}\right\}$ is dense in $W_{0}^{1, p^{+}}(\Omega)$, and the functions $u_{k}^{(m)}$ are solutions of problem (3.5). By this reason, to prove Theorem 6.1 it suffices to derive estimate (6.3) for the approximate solutions $u^{(m)}$.

For the sake of simplicity, throughout the proof we use the notation $u$ for the approximate solution $u^{(m)}$. Fix some $m$, multiply relations (3.5) by $u_{k, t}^{(m)}$ and take the sum over $k=1, \ldots, m$. This gives the equality

$$
\begin{aligned}
\left\|u_{t}\right\|_{2, \Omega}^{2} & +\sum_{i=1}^{n} \int_{\Omega}\left(a_{i}\left|D_{i} u\right|^{p_{i}-2} D_{i} u D_{i} u_{t}\right) d x \\
& +\int_{\Omega} c|u|^{\sigma-2} u u_{t} d x=\int_{\Omega} f u_{t} d x .
\end{aligned}
$$

We will use the easily verified formulas

$$
\begin{aligned}
a_{i}\left|D_{i} u\right|^{p_{i}-2} D_{i} u D_{i} u_{t}= & \frac{\partial}{\partial t}\left(a_{i} \frac{\left|D_{i} u\right|^{p_{i}}}{p_{i}}\right) \\
& +a_{i}\left|D_{i} u\right|^{p_{i}}\left(\frac{1}{p_{i}^{2}}-\frac{\ln \left|D_{i} u\right|^{p_{i}}}{p_{i}}\right) p_{i t}-a_{i t} \frac{\left|D_{i} u\right|^{p_{i}}}{p_{i}} \\
c|u|^{\sigma-2} u u_{t}= & \frac{\partial}{\partial t}\left(c \frac{|u|^{\sigma}}{\sigma}\right)+c|u|^{\sigma}\left(\frac{1}{\sigma^{2}}-\frac{\ln |u|}{\sigma}\right) \sigma_{t}-c_{t} \frac{|u|^{\sigma}}{\sigma}
\end{aligned}
$$


Using them in (6.4), we obtain the equality

$$
\left\|u_{t}\right\|_{2, \Omega}^{2}+Y^{\prime}(t)=I_{1}+\sum_{i=1}^{n} I_{2, i}+\sum_{i=1}^{n} I_{3, i}
$$

where

(6.6) $Y(t)=\int_{\Omega}\left(\sum_{i=1}^{n} a_{i} \frac{\left|D_{i} u\right|^{p_{i}}}{p_{i}}+c \frac{|u|^{\sigma}}{\sigma}\right) d x, \quad I_{1}=-\int_{\Omega} f u_{t} d x$,

(6.7) $\quad I_{2, i}=\int_{\Omega}\left(-a_{i}\left|D_{i} u\right|^{p_{i}}\left(\frac{1}{p_{i}^{2}}-\frac{\ln \left|D_{i} u\right|^{p_{i}}}{p_{i}}\right) p_{i t}+a_{i t} \frac{\left|D_{i} u\right|^{p_{i}}}{p_{i}}\right) d x$,

(6.8) $I_{3, i}=\int_{\Omega}\left(-c|u|^{\sigma}\left(\frac{1}{\sigma^{2}}-\frac{\ln |u|^{\sigma}}{\sigma}\right) \sigma_{t}+c_{t} \frac{|u|^{\sigma}}{\sigma}\right) d x$.

The following estimates hold:

$$
\begin{gathered}
\left|I_{1}\right| \leq \frac{\delta}{2}\left\|u_{t}\right\|_{2, \Omega}^{2}+\frac{1}{2 \delta}\|f\|_{2, \Omega}^{2}, \quad \delta \in(0,1), \\
\left.\left.\left|\int_{\Omega} a_{i}\right| D_{i} u\right|^{p_{i}} \frac{p_{i t}}{p_{i}^{2}} d x\left|\leq \max _{\bar{\Omega}}\right| p_{i t}\left|\int_{\Omega} a_{i}\right| D_{i} u\right|^{p_{i}} \frac{1}{p_{i}^{2}} d x=\lambda_{p}(t) \int_{\Omega} a_{i}\left|D_{i} u\right|^{p_{i}} \frac{1}{p_{i}^{2}} d x, \\
\int_{\Omega} a_{i}\left|D_{i} u\right|^{p_{i}}\left(\frac{\ln \left|D_{i} u\right|^{p_{i}}}{p_{i}}\right) p_{i t} d x \equiv A+B, \\
A=-\int_{\Omega \cap\left(\left|D_{i} u\right|>1\right)}\left|p_{i t}\right| a_{i}\left|D_{i} u\right|^{p_{i}} \ln \left|D_{i} u\right| d x=-|A| \leq 0
\end{gathered}
$$

(recall that $p_{i t} \leq 0, \ln \left|D_{i} u\right|^{p_{i}}>0$ )

$$
\begin{gathered}
|B|=\int_{\Omega \cap\left\{\left|D_{i} u\right| \in[0,1]\right\}} \cdots \leq a_{1}|\Omega| \max _{\bar{\Omega}}\left|p_{i t}\right| \max _{\tau \in[0,1]}\left|\tau^{p_{i}} \ln \tau\right| \leq C \lambda_{p}(t), \\
\left|\int_{\Omega} a_{i t} \frac{\left|D_{i} u\right|^{p_{i}}}{p_{i}} d x\right| \leq \lambda_{a}(t) \int_{\Omega} \frac{\left|D_{i} u\right|^{p_{i}}}{p_{i}} d x .
\end{gathered}
$$


Gathering these estimate we obtain the inequality

$$
\begin{aligned}
I_{2} \leq & -\sum_{i=1}^{n} \int_{\Omega}\left|p_{i t}\right| a_{i}\left|D_{i} u\right|^{p_{i}}|\ln | D_{i} u|| d x+C \lambda_{p}(t) \\
& +\lambda_{p}(t) \sum_{i=1}^{n} \int_{\Omega} a_{i}\left|D_{i} u\right|^{p_{i}} \frac{1}{p_{i}^{2}} d x+\lambda_{a}(t) \sum_{i=1}^{n} \int_{\Omega} \frac{\left|D_{i} u\right|^{p_{i}}}{p_{i}} d x \\
\leq & -\sum_{i=1}^{n} \int_{\Omega}\left|p_{i t}\right| a_{i}\left|D_{i} u\right|^{p_{i}}|\ln | D_{i} u|| d x+C\left[\lambda_{p}(t)+\lambda_{a}(t)\right] Y(t)+C \lambda_{p}(t) .
\end{aligned}
$$

The terms $I_{3, i}$ are estimated likewise:

$$
\begin{gathered}
\left.\left|\int_{\Omega} c\right| u\right|^{\sigma} \frac{\sigma_{t}}{\sigma^{2}} d x \mid \leq C \lambda_{\sigma}(t) Y(t), \\
-\int_{\Omega \cap\left(\left|u^{N}\right|>1\right)} c|u|^{\sigma} \ln |u|^{\sigma}\left|\sigma_{t}\right| d x+\int_{\Omega \cap\left(\left|u^{N}\right| \leq 1\right)} c|u|^{\sigma} \ln |u|^{\sigma}\left|\sigma_{t}\right| d x \\
\leq-\int_{\Omega} \sum_{i=1}^{n}\left(\left.c|u|^{\sigma}|\ln | u\right|^{\sigma}|| \sigma_{t} \mid\right) d x+C \lambda_{\sigma}(t), \\
\left|\int_{\Omega} \sum_{i=1}^{n} c_{t} \frac{|u|^{\sigma}}{\sigma} d x\right| \leq C \lambda_{c}(t) Y(t) .
\end{gathered}
$$

It follows that

$$
I_{3} \leq-\int_{\Omega} \sum_{i=1}^{n}\left(c|u|^{\sigma} \ln |u|^{\sigma}\left|\sigma_{t}\right|\right) d x+C\left(\lambda_{c}(t)+\lambda_{\sigma}(t)\right) Y(t)+C \lambda_{\sigma}(t) .
$$

Then the function $Y(t)$ satisfies the differential inequality

$$
\begin{aligned}
Y^{\prime}(t) & +\left\|u_{t}\right\|_{2, \Omega}^{2} \\
& +\sum_{i=1}^{n} \int_{\Omega}\left(c|u|^{\sigma} \ln |u|^{\sigma}\left|\sigma_{t}\right|\right) d x \\
& +\sum_{i=1}^{n} \int_{\Omega}\left|p_{i t}\right| a_{i}\left|D_{i} u\right|^{p_{i}}|\ln | D_{i} u \mid d x \leq C[\Lambda(t) Y(t)+\lambda(t)+1]
\end{aligned}
$$

with

$$
\Lambda(t)=\lambda_{p}(t)+\lambda_{a}(t)+\lambda_{c}(t)+\lambda_{\sigma}(t), \quad \lambda(t)=\lambda_{\sigma}(t)+\lambda_{p}(t),
$$

and the assertion follows from Gronwall's lemma. 
Remark 6.1. The assertion remains true for the solutions of the equation

$$
u_{t}-\sum_{i=1}^{n}\left[\frac{d}{d x_{i}}\left(a_{i}(z)\left|D_{i} u\right|^{p_{i}(z)-2} D_{i} u\right)+c_{i}(z)|u|^{\sigma_{i}(z)-2} u\right]=f(z),
$$

provided that the functions $c_{i}(z), \sigma_{i}(z)$ satisfy the conditions of Theorem 6.1.

Remark 6.2. Problem (6.1) includes, as a partial case, the problem

$$
\left\{\begin{array}{l}
u_{t}=\Delta_{p} u+f \quad \text { in } Q_{T}, \\
u=0 \text { on } \Gamma_{T}, u(x, 0)=u_{0}(x) \text { in } \Omega,
\end{array}\right.
$$

with constant $p \in(1, \infty)$. It was proved in $[\mathbf{1 9}$, Lemma 2.1] that the solutions of this problem satisfy the estimate

(6.11) $\left\|u_{t}\right\|_{L^{2}\left(Q_{T}\right)}^{2}+\|u\|_{L^{\infty}\left(0, T ; W^{1, p}(\Omega)\right)}^{p} \leq C\left(\|f\|_{L^{2}\left(Q_{T}\right)}^{2}+\left\|u_{0}\right\|_{W^{1, p}(\Omega)}^{p}\right)$,

which is contained in (6.3) if $p=$ const. Moreover, if in the conditions of Theorem 6.1 the coefficients $a_{i}, c$ and the exponents $p_{i}$ and $\sigma$ are variable but independent of $t$, then estimate (6.11) is true as well for the solutions of problem (6.1).

\section{Extensions}

The results of this paper can be extended in various directions. Let us mention here several most obvious generalizations.

1. The class of equations (1.1) can be completed by the equations

$$
\begin{aligned}
u_{t} & -\sum_{i} \frac{d}{d x_{i}}\left[a_{i}(z, u)\left|D_{i} u\right|^{p_{i}(z)-2} D_{i} u+b_{i}(z, u)\right] \\
& +\sum_{i} d_{i}(z, u) D_{i} u+d(z, u)=0
\end{aligned}
$$

which reduce to (1.1) by means of the substitution

$\widetilde{b}_{i}(z, u) \equiv b_{i}(z, u)+\int_{0}^{u} d_{i}(z, s) d s, \quad \widetilde{d}(z, u) \equiv d(z, u)-\sum_{i} \int_{0}^{u} D_{i} d_{i}(z, s) d s$.

2. The main existence Theorem 3.1 remains true if in the growth conditions (1.4) $h_{d}(z) \in \mathbf{W}^{\prime}\left(Q_{T}\right)$, i.e.,

$$
\begin{cases}h_{d}(z)=h_{d}^{0}(z)+\operatorname{div} H(z), & h_{d}^{0} \in L^{\lambda^{\prime}}\left(Q_{T}\right), \\ H(z)=\left(H_{1}, \ldots, H_{n}\right), & H_{i} \in L^{p_{i}^{\prime}(\cdot)}\left(Q_{T}\right) .\end{cases}
$$


3. The proofs of the main theorems can be easily adapted to the equations

$$
u_{t}-\sum_{i} \frac{d}{d x_{i}}\left[a_{i}(z, u)|\nabla u|^{p(z)-2} D_{i} u+b_{i}(z, u)\right]+d(z, u)=0 .
$$

For the main function spaces we take

$$
\begin{gathered}
\mathbf{V}_{t}(\Omega)=\left\{\left.u(x)\left|u(x) \in L^{2}(\Omega) \cap W_{0}^{1,1}(\Omega),\right| \nabla u(x)\right|^{p(x, t)} \in L^{1}(\Omega)\right\}, \\
\|u\|_{\mathbf{V}_{t}(\Omega)}=\|u\|_{2, \Omega}+\|\nabla u\|_{p(\cdot, t), \Omega},
\end{gathered}
$$

and

$$
\begin{gathered}
\mathbf{W}\left(Q_{T}\right)=\left\{u:[0, T] \mapsto \mathbf{V}_{t}(\Omega)\left|u \in L^{2}\left(Q_{T}\right),\right| \nabla u \mid \in L^{p(\cdot)}\left(Q_{T}\right), u=0 \text { on } \Gamma\right\} \\
\|u\|_{\mathbf{W}\left(Q_{T}\right)}=\|\nabla u\|_{p(\cdot), Q_{T}}+\|u\|_{2, Q_{T}} .
\end{gathered}
$$

The rest of the arguments does not need any change.

4. The proofs of the existence theorems can be adapted to the case of the Neumann boundary condition. For example, let us consider the problem

$$
\begin{cases}u_{t}-\sum_{i} \frac{d}{d x_{i}}\left(a_{i}(z, u)\left|D_{i} u\right|^{p_{i}(z)-2} D_{i} u\right)+d(z, u)=0 & \text { in } Q_{T} \\ \sum_{i} a_{i}(z, u)\left|D_{i} u\right|^{p_{i}(z)-2} D_{i} u \cdot \nu_{i}=0 & \text { on } \Gamma_{T} \\ u(x, 0)=u_{0}(x) & \text { in } \Omega,\end{cases}
$$

where $\nu=\left(\nu_{1}, \ldots, \nu_{n}\right)$ denotes the outer normal vector to $\Gamma_{T}$. Let us introduce the function spaces

$$
\begin{gathered}
\mathbf{V}_{t}(\Omega)=\left\{u(x): u(x) \in L^{2}(\Omega),\left|D_{i} u(x)\right|^{p_{i}(x, t)} \in L^{1}(\Omega)\right\}, \\
\mathbf{W}\left(Q_{T}\right)=\left\{u:\left.[0, T] \mapsto \mathbf{V}_{t}(\Omega)\left|u \in L^{2}\left(Q_{T}\right),\right| D_{i} u\right|^{p_{i}(z)} \in L^{1}\left(Q_{T}\right)\right\}
\end{gathered}
$$

with the norms

$$
\begin{aligned}
& \|u\|_{\mathbf{V}_{t}(\Omega)}=\|u\|_{2, \Omega}+\sum_{i}\left\|D_{i} u\right\|_{p_{i}(\cdot, t), \Omega}, \\
& \|u\|_{\mathbf{W}\left(Q_{T}\right)}=\sum_{i}\left\|D_{i} u\right\|_{p_{i}(\cdot), Q_{T}}+\|u\|_{2, Q_{T}} .
\end{aligned}
$$


We say that function $u(x, t) \in \mathbf{W}\left(Q_{T}\right) \cap L^{\infty}\left(0, T ; L^{2}(\Omega)\right)$ is a weak solution of problem (7.1) if for every test-function

$$
\zeta \in \mathbf{Z} \equiv\left\{\eta(z): \eta \in \mathbf{W}\left(Q_{T}\right) \cap L^{\infty}\left(0, T ; L^{2}(\Omega)\right), \eta_{t} \in \mathbf{W}^{\prime}\left(Q_{T}\right)\right\},
$$

and every $t_{1}, t_{2} \in[0, T]$ the following identity holds:

$$
\int_{t_{1}}^{t_{2}} \int_{\Omega}\left(u \zeta_{t}-\sum_{i} a_{i}\left|D_{i} u\right|^{p_{i}-2} D_{i} u D_{i} \zeta-d(z, u) \zeta\right) d z=\left.\int_{\Omega} u \zeta d x\right|_{t_{1}} ^{t_{2}}
$$

Let us assume that the data of problem (7.1) satisfy the conditions of Theorem 3.1. Since the space $\mathbf{W}\left(Q_{T}\right)$ is separable, a solution of problem (7.1) can be constructed as the limit of the sequence of Galerkin's approximations (see the proof of Theorem 3.1).

\section{References}

[1] E. Acerbi and G. Mingione, Regularity results for a class of functionals with non-standard growth, Arch. Ration. Mech. Anal. 156(2) (2001), 121-140.

[2] E. Acerbi and G. Mingione, Regularity results for electrorheological fluids: the stationary case, C. R. Math. Acad. Sci. Paris 334(9) (2002), 817-822.

[3] E. Acerbi And G. Mingione, Regularity results for stationary electro-rheological fluids, Arch. Ration. Mech. Anal. 164(3) (2002), 213-259.

[4] E. Acerbi, G. Mingione, and G. A. Seregin, Regularity results for parabolic systems related to a class of non-Newtonian fluids, Ann. Inst. H. Poincaré Anal. Non Linéaire 21(1) (2004), 25-60.

[5] H. W. Alt and S. Luckhaus, Quasilinear elliptic-parabolic differential equations, Math. Z. 183(3) (1983), 311-341.

[6] L. Álvarez, P.-L. Lions, and J.-M. Morel, Image selective smoothing and edge detection by nonlinear diffusion. II, SIAM J. Numer. Anal. 29(3) (1992), 845-866.

[7] F. Andreu, V. Caselles, and J. M. Mazón, The Cauchy problem for a strongly degenerate quasilinear equation, J. Eur. Math. Soc. (JEMS) 7(3) (2005), 361-393.

[8] F. Andreu, V. Caselles, and J. M. Mazón, "Parabolic quasilinear equations minimizing linear growth functionals", Progress in Mathematics 223, Birkhäuser Verlag, Basel, 2004. 
[9] S. Antontsev and M. Chipot, Anisotropic equations: uniqueness and existence results, Differential Integral Equations 21(5-6) (2008), 401-419.

[10] S. Antontsev, M. Chipot, And Y. Xie, Uniqueness results for equations of the $p(x)$-Laplacian type, Adv. Math. Sci. Appl. 17(1) (2007), 287-304.

[11] S. N. Antontsev and J. F. Rodrigues, On stationary thermorheological viscous flows, Ann. Univ. Ferrara Sez. VII Sci. Mat. 52(1) (2006), 19-36.

[12] S. N. Antontsev and S. I. Shmarev, A model porous medium equation with variable exponent of nonlinearity: existence, uniqueness and localization properties of solutions, Nonlinear Anal. 60(3) (2005), 515-545.

[13] S. N. Antontsev and S. I. Shmarev, Elliptic equations and systems with nonstandard growth conditions: existence, uniqueness and localization properties of solutions, Nonlinear Anal. 65(4) (2006), 728-761.

[14] S. N. Antontsev and S. I. Shmarev, Elliptic equations with anisotropic nonlinearity and nonstandard growth conditions, in: "Handbook of Differential Equations: stationary partial differential equations" (M. Chipot and P. Quittner, eds.), vol. 3, Elsevier, 2006, pp. $1-100$.

[15] S. N. Antontsev and S. I. Shmarev, Parabolic equations with anisotropic nonstandard growth conditions, in: "Free boundary problems", Internat. Ser. Numer. Math. 154, Birkhäuser, Basel, 2007, pp. 33-44.

[16] S. N. Antontsev and S. I. Shmarev, Existence and uniqueness of solutions of degenerate parabolic equations with variable exponents of nonlinearity, (Russian), Fundam. Prikl. Mat. 12(4) (2006), 3-19; translation in: J. Math. Sci. (N. Y.) 150(5) (2008), 2289-2301.

[17] S. N. Antontsev and S. I. Shmarev, Extinction of solutions of parabolic equations with variable anisotropic nonlinearities, Proc. Steklov Inst. Math. 261(1) (2008), 11-21.

[18] S. Antontsev And V. Zhikov, Higher integrability for parabolic equations of $p(x, t)$-Laplacian type, Adv. Differential Equations 10(9) (2005), 1053-1080.

[19] J. W. BARRETT AND W. B. LiU, Finite element approximation of the parabolic p-Laplacian, SIAM J. Numer. Anal. 31(2) (1994), $413-428$. 
[20] L. Boccardo, T. Gallouët, and J. L. VÁzquez, Solutions of nonlinear parabolic equations without growth restrictions on the data, Electron. J. Differential Equations 60 (2001), 20 pp. (electronic).

[21] Y. Chen, S. Levine, and M. RaO, Variable exponent, linear growth functionals in image restoration, SIAM J. Appl. Math. 66(4) (2006), 1383-1406 (electronic).

[22] M. Chipot and G. Michaille, Uniqueness results and monotonicity properties for strongly nonlinear elliptic variational inequalities, Ann. Scuola Norm. Sup. Pisa Cl. Sci. (4) 16(1) (1989), $137-166$.

[23] M. Chipot and G. Michaille, Uniqueness results and monotonicity properties for the solutions of some variational inequalities. Existence of a free boundary, in: "Free boundary problems: theory and applications", Vol. I (Irsee, 1987), Pitman Res. Notes Math. Ser. 185, Longman Sci. Tech., Harlow, 1990, pp. 271-276.

[24] E. DiBenedetto, "Degenerate parabolic equations", Universitext, Springer-Verlag, New York, 1993.

[25] L. Diening, Maximal function on generalized Lebesgue spaces $L^{p(\cdot)}$, Math. Inequal. Appl. 7(2) (2004), 245-253.

[26] D. E. Edmunds ANd J. RÁKOSNík, Sobolev embeddings with variable exponent, Studia Math. 143(3) (2000), 267-293.

[27] F. EtTwein And M. RỦžičKa, Existence of strong solutions for electrorheological fluids in two dimensions: steady Dirichlet problem, in: "Geometric analysis and nonlinear partial differential equations", Springer, Berlin, 2003, pp. 591-602.

[28] P. Harjulehto and P. Hästö, An overview of variable exponent Lebesgue and Sobolev spaces, in: "Future trends in geometric function theory", Rep. Univ. Jyväskylä Dep. Math. Stat. 92, Univ. Jyväskylä, Jyväskylä, 2003, pp. 85-93.

[29] J. KAČUR, On a solution of degenerate elliptic-parabolic systems in Orlicz-Sobolev spaces. II, Math. Z. 203(4) (1990), 569-579.

[30] A. S. Kalashnikov, Some problems of the qualitative theory of second-order nonlinear degenerate parabolic equations, (Russian), Uspekhi Mat. Nauk 42 (1987), no. 2(254), 135-176, 287.

[31] A. S. KALAShnikov, Nonlinear phenomena in nonstationary processes described by asymptotically linear equations, (Russian), Differentsial'nye Uravneniya 29(3) (1993), 381-391, 549; translation in: Differential Equations 29(3) (1993), 324-334.

[32] A. S. Kalashnikov, Perturbation of critical exponents in some nonlinear problems of mathematical physics, (Russian), Dokl. Akad. 
Nauk 337(3) (1994), 320-322; translation in: Phys. Dokl. 39(7) (1994), 474-476.

[33] A. S. Kalashnikov, On some nonlinear problems in mathematical physics with exponents that are close to critical, (Russian), Tr. Semin. im. I. G. Petrovskogo 19 (1996), 73-98, 346; translation in: J. Math. Sci. (New York) 85(6) (1997), 2287-2301.

[34] O. KovÁČIK AND J. RÁKOSNÍK, On spaces $L^{p(x)}$ and $W^{k, p(x)}$, Czechoslovak Math. J. 41(116) (1991), no. 4, 592-618.

[35] A. Kufner, J. OldřIch, AND S. Fučík, "Function spaces", Monographs and Textbooks on Mechanics of Solids and Fluids; Mechanics: Analysis, Noordhoff International Publishing, Leyden, Academia, Prague, 1977.

[36] J.-L. Lions, "Quelques méthodes de résolution des problèmes aux limites non linéaires", Dunod, Gauthier-Villars, Paris, 1969.

[37] G. Mingione, Regularity of minima: an invitation to the dark side of the calculus of variations, Appl. Math. 51(4) (2006), 355-426.

[38] J. Musielak, "Orlicz spaces and modular spaces", Lecture Notes in Mathematics 1034, Springer-Verlag, Berlin, 1983.

[39] K. R. RAJAgopal AND M. RÚŽIČKA, Mathematical modeling of electrorheological materials, Contin. Mech. Thermodyn. 13(1) (2001), 59-78.

[40] M. RÚŽIČKA, "Electrorheological fluids: modeling and mathematical theory", Lecture Notes in Mathematics 1748, Springer-Verlag, Berlin, 2000.

[41] S. G. SAmko, On a progress in the theory of Lebesgue spaces with variable exponent: maximal and singular operators, Integral Transforms Spec. Funct. 16(5-6) (2005), 461-482.

[42] S. G. SAmko, Density $C_{0}^{\infty}\left(R^{n}\right)$ in the generalized Sobolev spaces $W^{m, p(x)}\left(R^{n}\right)$, (Russian), Dokl. Akad. Nauk 369(4) (1999), 451-454.

[43] J. Simon, Compact sets in the space $L^{p}(0, T ; B)$, Ann. Mat. Pura Appl. (4) 146 (1987), 65-96.

[44] M. YU AND X. Lian, Boundedness of solutions of parabolic equations with anisotropic growth conditions, Canad. J. Math. 49(4) (1997), 798-809.

[45] V. V. Zhikov, On the density of smooth functions in SobolevOrlicz spaces, (Russian), Zap. Nauchn. Sem. S.-Peterburg. Otdel. Mat. Inst. Steklov. (POMI) 310 (2004), Kraev. Zadachi Mat. Fiz. i Smezh. Vopr. Teor. Funkts. 35 [34], 67-81, 226; translation in: J. Math. Sci. (N. Y.) 132(3) (2006), 285-294. 
S. Antontsev:

Centro de Matemática e Aplicações Fundamentais (CMAF)

Universidade de Lisboa

1649-003 Lisboa

Portugal

E-mail address: antontsevsn@mail.ru

S. Shmarev:

Departamento de Matemáticas

Universidad de Oviedo

33071 Oviedo

Spain

E-mail address: shmarev@orion.ciencias.uniovi.es

Primera versió rebuda el 14 de març de 2008, darrera versió rebuda el 18 de setembre de 2008 . 